\title{
Current Status of the Facility Instrumentation Suite at The Large Binocular Telescope Observatory
}

\author{
Barry Rothberg ${ }^{\mathrm{a}}$, Olga Kuhn ${ }^{\mathrm{a}}$, Michelle L. Edwards ${ }^{\mathrm{a}}$, John M. Hill ${ }^{\mathrm{a}}$, David Thompson ${ }^{\mathrm{a}}$, \\ Christian Veillet ${ }^{\mathrm{a}}$, and R. Mark Wagner ${ }^{\mathrm{a}}$

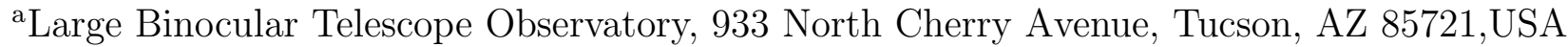

\begin{abstract}
The current status of the facility instrumentation for the Large Binocular Telescope (LBT) is reviewed. The LBT encompasses two 8.4 meter primary mirrors on a single mount yielding an effective collecting area of 11.8 meters or 23 meters when interferometrically combined. The three facility instruments at LBT include: 1) the Large Binocular Cameras (LBCs), each with a $23^{\prime} \times 25^{\prime}$ field of view (FOV). The blue optimized and red optimized optical wavelength LBCs are mounted at the prime focus of the SX (left) and DX (right) primary mirrors, respectively. Combined, the filter suite of the two LBCs cover 0.3-1.1 $\mu \mathrm{m}$, including the addition of new medium-band filters centered on $\mathrm{TiO}(0.78 \mu \mathrm{m})$ and $\mathrm{CN}(0.82 \mu \mathrm{m}) ; 2)$ the Multi-Object Double Spectrograph (MODS), two identical optical spectrographs each mounted at the straight through f/15 Gregorian focus of the primary mirrors. The capabilities of MODS- 1 and -2 include imaging with Sloan filters $(u, g, r, i$, and $z)$ and medium resolution $(R \sim 2000)$ spectroscopy, each with 24 interchangeable masks (multi-object or longslit) over a $6^{\prime} \times 6^{\prime}$ FOV. Each MODS is capable of blue $(0.32-0.6 \mu \mathrm{m})$ and red $(0.5-1.05 \mu \mathrm{m})$ wavelength only spectroscopy coverage or both can employ a dichroic for 0.32-1.05 $\mu \mathrm{m}$ wavelength coverage (with reduced coverage from 0.56$0.57 \mu \mathrm{m}$ ); and 3) the two LBT Utility Camera in the Infrared instruments (LUCIs), are each mounted at a bent-front Gregorian f/15 focus of a primary mirror. LUCI-1 \& 2 are designed for seeing-limited $\left(4^{\prime} \times 4^{\prime}\right.$ FOV $)$ and active optics using thin-shell adaptive secondary mirrors $\left(0.5^{\prime} \times 0.5^{\prime} \mathrm{FOV}\right)$ imaging and spectroscopy over the wavelength range of 0.95-2.5 $\mu \mathrm{m}$ and spectroscopic resolutions of $400 \leq R \leq 11000$ (depending on the combination of grating, slits, and cameras used). The spectroscopic capabilities also include 32 interchangeable multi-object or longslit masks which are cryogenically cooled. Currently all facility instruments are in-place at the LBT and, for the first time, have been on-sky for science observations. In Summer 2015 LUCI-1 was refurbished to replace the infrared detector; to install a high-resolution camera to take advantage of the active optics SX secondary; and to install a grating designed primarily for use with high resolution active optics. Thus, like MODS- $1 \&-2$, both LUCIs now have specifications nearly identical to each other. The software interface for both LUCIs have also been replaced, allowing both instruments to be run together from a single interface. With the installation of all facility instruments finally complete we also report on the first science use of "mixed-mode" operations, defined as the combination of different paired instruments with each mirror (i.e. LBC+MODS, LBC+LUCI, LUCI+MODS). Although both primary mirrors reside on a single fixed mount, they are capable of operating as independent entities within a defined "co-pointing" limit. This provides users with the additional capability to independently dither each mirror or center observations on two different sets of spatial coordinates within this limit.
\end{abstract}

Keywords: ELT, Observatories, Instrumentation, Binocular, Spectroscopy, Imaging

\section{INTRODUCTION}

The Large Binocular Telescope Observatory (LBTO) is situated near the city of Safford in southeastern Arizona in the Pinaleño Mountains. It is part of the Mount Graham International Observatory (MGIO) located on Emerald Peak on the highest mountain, Mount Graham. LBTO sits at an altitude of 3192 meters. As the name implies, the Large Binocular Telescope (LBT) houses two primary mirrors, separated by 14.4 meters (center-to-center), mounted on a single altitude-azimuth mount. Each mirror is 8.4 meters in diameter, with a

Further author information: (Send correspondence to Barry Rothberg)

E-mail: brothberg@lbto.org, Telephone: +1 520 626-8672, Fax: +1 520 626-9333 
combined collecting area equivalent to a single 11.8 meter mirror, or an interferometric baseline of 22.65 meters, edge-to-edge. Although the LBT is comprised of two 8.4 meter mirrors, their fast $f / 1.14$ focal ratio allows for a compact mount and co-rotating enclosure, see Hill et al. (2004)!1 Ashby et al. (2006) ${ }^{\frac{1}{2}}$ Hill et al. (2006) ${ }^{3]}$ and Hill et al. (2010 $)^{4}$ for more details. The binocular design is combined with four Bent Gregorian focal stations (three with instrument rotator bearings) and one Direct Gregorian focal station for each side of the telescope. The two mirrors can be used in binocular mode with the same pairs of instruments as well as each independently with different instrumentation. Switching between different optical instrumentation is done by moving various swing arms which hold the prime focus optical cameras, or secondary and tertiary mirrors. The transition between prime focus and Gregorian instruments takes $\sim 20$ minutes, while transitions between different Gregorian instruments can take $\leq 10$ minutes. This flexibility is advantageous for incorporating a variety of scientific programs during a single night as well as adapting quickly to changes in site and weather conditions. For brevity, the left-side of the telescope is denoted as $S X$ and the right-side is denoted as $D X$.

The LBT is an international partnership which includes the University of Arizona (25\%) including access for Arizona State University and Northern Arizona University; Germany (25\%) or LBTB (Beteiligungsgesellschaft) which includes participation of five German institutes (Landessternwarte Königstuhl, Leibniz Institute for Astrophysics Potsdam, Max-Planck-Institut für Astronomie, Max-Planck-Institut für Extraterrestrische Physik, and Max-Planck-Institut für Radioastronomie); Italy (25\%) or Instituto Nazionale di Astrofisica which is responsible for offering access to the Italian community to LBTO; the Ohio State University (12.5\%); and the Research Corporation for Science and Advancement (RC) which coordinates the participation of four universities (Ohio State University, University of Notre Dame, University of Minnesota, and University of Virginia).

In 2014, the second Multi-Object Double Spectrograph (MODS-2) was installed on the LBT. This marked the completion of the installation of all facility instrumentation. Beginning with the 2014B semester and up through the 2016A semester, all facility instruments (3 on SX, 3 on DX) were available for on-sky scientific use during partner and LBTO science time or underwent commissioning (or re-commissioning). For the 2016B semester, all six facility instruments will be available for on-sky scientific use by the LBTO and its partners. In this conference proceeding, we present a summary of the LBT scientific facility instruments that are now available for partner science observations. It is an update on the significant changes that have occurred at LBTO since Wagner et al. $(2014) \cdot[$

\section{TYPES OF INSTRUMENTATION}

There are three categories of LBT scientific instrumentation. The first are facility instruments, which are available for use by anyone within the partnerships. Facility instruments are supported and maintained by LBTO personnel. Although during commissioning phases, facility instruments are still supported by the instrument teams, who work in conjunction with LBTO staff. The second are Principal Investigator instruments such as the Potsdam Echelle and Polarimetric Spectroscopic Instrument (PEPSI), which uses both primary mirrors, see Strassmeier et al. (2008) ${ }^{\sqrt{6}}$ for more information, and has been used on-sky for scientific observations during the 2015B and 2016A semesters. These are maintained and operated solely by the builders, but may be used by LBTO partners for science on a collaborative basis or through time exchanges at the discretion of the instrument principal investigator (PI). LBTO provides limited technical assistance to enable the instruments to interface with the telescope control systems and telescope infrastructure. The third type are Strategic instruments, which are technically challenging and designed to push the limits of astronomical instrumentation. They may be uniquely suited to the LBT and are designed to have a major impact on astronomy. Strategic instruments may be available to the LBT community on a collaborative basis or through time exchanges. A current strategic instrument is the LBT Interferometer (LBTI), which uses both primary mirrors and comprises LMIRCam (3-5 $\mu \mathrm{m})$ and the NOMIC $(8-13 \mu \mathrm{m})$ camera. They are currently operational for on-sky scientific observations, see Hinz et al. (2008) ${ }^{7}$ Wilson et al. (2008), ${ }^{8}$ Skrutskie et al. 2010, ${ }^{9}$ Leisenring et al. (2012) ${ }^{10}$ and Hoffmann et al. $(2014)^{111}$ for more information. Recent results include mapping the $5 \mu \mathrm{m}$ emission of the Loki Patera volcanoes on Jupiter's moon Io using the SX and DX mirrors in interferometric mode (Conrad et al. 2015 $5^{12}$ ) or the LBTI Exozodi Exoplanet Common Hunt (LEECH) survey (Skemer et al. 2016 $6^{13}$ ). Future strategic instruments include: LBT INterferometric Camera and the NearIR/Visible Adaptive iNterferometer for Astronomy (LINCNIRVANA), a multi-conjugate adaptive optics (MCAO) near-IR imaging system that provides both ground-layer and high-layer corrections. It is in the first stages of on-telescope testing (Gassler et al. (2004), $\frac{14}{14}$ Herbst et al. 
$\left.(2014)^{15}\right)$; and iLocater, a diffraction-limited Doppler spectrometer with high spectral resolution $(R \sim 110,000)$ operating in the $Y$-band that will be used to characterize Earth-like exo-planets orbiting M-dwarf stars. iLocater will use a fiber-fed AO-corrected beam to pass light to a compact spectrograph (Crepp at al. 2014, $\frac{16}{,}$ Veillet et al. 2014 ${ }^{17}$ ). Further discussion of PI and Strategic instruments are beyond the scope of this paper.

\section{FACILITY INSTRUMENTS}

The Large Binocular Cameras (LBCs) are a pair of blue- and red-optimized prime focus imagers with a field of view just shy the size of the Moon projected on the sky. The LBC filter suite covers $U$-band $(0.33 \mu \mathrm{m})$ in the blue through $Y$-band $(1.1 \mu \mathrm{m})$ in the red. The Multi-Object Double Spectrographs 1 and 2 (MODS-1 and MODS-2) are a pair of optical spectrographs (longslit and custom located at the direct Gregorian foci at each primary mirror. The two LBT NIR Spectroscopic Utility with Camera Instruments (LUCIs) are capable of imaging and spectroscopy (longslit and custom designed multi-object slit masks) each located at one of the Bent f/15 Gregorian ports. As of the 2016A semester all facility instruments have been used for scientific observations on-sky. Table 1 presents a brief overview of the scientific capabilities of the facility instruments. Specific details will be discussed or cited in subsequent sections.

Table 1. Overview of Facility Instruments \& Capabilities

\begin{tabular}{|c|c|c|c|c|c|c|}
\hline Instrument & $\begin{array}{l}\text { Focal } \\
\text { Station }\end{array}$ & MODES & $\begin{array}{l}\lambda \text { Range } \\
(\mu \mathrm{m})\end{array}$ & $\begin{array}{l}\text { FOV } \\
\left({ }^{\prime}\right)\end{array}$ & $\begin{array}{l}\text { Plate Scale } \\
(" / \text { pixel })\end{array}$ & Resolution \\
\hline LBC Blue & SX Prime & Imaging & $0.33-0.67$ & $23^{\prime} \times 25^{\prime}$ & 0.2255 & $\ldots$ \\
\hline LBC Red & DX Prime & Imaging & $0.55-1.11$ & $23^{\prime} \times 25^{\prime}$ & 0.2255 & $\ldots$ \\
\hline MODS-1/-2 & $\begin{array}{l}f / 15 \text { Direct } \\
\text { Gregorian }\end{array}$ & $\begin{array}{l}\text { Imaging } \\
\text { Spectroscopy }\end{array}$ & $0.31-1.1$ & $6^{\prime} \times 6^{\prime}$ & $\begin{array}{l}0.120 \text { (blue) } \\
0.123 \text { (red) }\end{array}$ & $\begin{array}{l}2300 \text { (blue) } \\
1850 \text { (red) } \\
\text { (100-500 prism) }\end{array}$ \\
\hline $\begin{array}{l}\text { LUCI-1/-2 } \\
\text { (Seeing Limited) }\end{array}$ & $\begin{array}{l}f / 15 \text { Bent } \\
\text { Greogorian }\end{array}$ & $\begin{array}{l}\text { Imaging } \\
\text { Spectroscopy }\end{array}$ & $0.95-2.5$ & $4^{\prime} \times 4^{\prime}$ & $\begin{array}{l}0.25(\mathrm{~N} 1.8) \\
0.12(\mathrm{~N} 3.75)\end{array}$ & $\begin{array}{l}1500-5500(\mathrm{~N} 1.8) \\
3000-11,000(\mathrm{~N} 3.75)\end{array}$ \\
\hline
\end{tabular}

\subsection{Large Binocular Cameras (LBCs)}

The LBCs are comprised of two wide-field imagers, one blue optimized at the $f / 1.14$ prime focus on SX, and one red optimized at the $f / 1.14$ prime focus on DX. They are each mounted on a spider swing-arm that can be deployed above their respective primary mirror and moved into and out of the telescope beam as required. The LBCs were accepted as facility instruments in October 2011. The two instruments were an in-kind contribution by INAF to the first generation of LBT instruments. Specific details regarding construction, commissioning, and upgrades can be found in Ragazzoni et al. (2006) ${ }^{18}$ Speziali et al. (2008) ${ }^{19]}$ and Giallongo et al. (2008)! $!^{20}$ The LBCs are the first instruments to make full use of the binocular capabilities of the LBT. Binocular observing has been done with the LBCs since the installation and commissioning of LBC Red was completed.

Owing to the fast focal ratio of the primary mirrors and placement at prime focus, a set of refractor corrector lenses is required to deal with geometric distortions that would affect the large field of view (FOV). Each LBC uses a similar set of five corrective lens (a 6th lens is the cryostat window with almost no net power). This is based on the Wynne approach of positive-negative-positive lenses (Wynne 1972 ${ }^{21}$ ), but with the second and third elements each split into two lenses. A filter wheel sits between the $5^{t h}$ and $6^{\text {th }}$ corrective lens (the first lens is defined as closest to the primary mirror). The lenses in LBC Blue are made of fused silica which permits better transmittance of light at shorter wavelengths $(0.3-0.5 \mu \mathrm{m})$. The lenses in LBC Red use borosilicate glass (BK7) and are optimized for longer wavelengths $(\lambda>0.5 \mu \mathrm{m})$. The corrected fields have a diameter of $110 \mathrm{~mm}$ and $108.2 \mathrm{~mm}$ for LBC Blue and LBC Red (equivalent to $27^{\prime}$ in diameter), respectively. The science detectors cover $\sim 75 \%$ of this area.

The LBCs each contain six E2V CCD detectors, four of which are used for science. The four science CCDs 
are E2V 420-90s with $2048 \times 4608(13.5 \mu$ m square pixels) are arranged in a mosaic with three abutted next to each other. A fourth CCD is rotated clockwise 90 degrees and centered along the top of the three science CCDs. Each CCD covers $7^{\prime} .8 \times 17^{\prime} .6$ with a gap of 70 pixels $\left(18^{\prime \prime}\right)$ between each CCD. This yields a $23^{\prime} \times 25^{\prime}$ FOV. In order to obtain an uninterrupted image, dithering is required to to fill the gaps between CCDs (and recommended to correct for cosmic rays and bad pixel columns). The un-binned readout time for the full array of science CCDs is 27 seconds. The other two CCDs are used for guiding and tracking collimation and wavefront control. They are E2V 420-90 custom made $512 \times 2048$ (13.5 $\mu$ m square) pixel CCDs that do not have a shutter mechanism. They are placed on either of the science CCD chips. One is within the focal plane and is used for guiding adjustments, the other is out of the focal plane and uses extra-focal pupil images to maintain collimation and focus. Figure 1 shows the layout of LBC Blue (LBC Red is the same layout with a slight difference in the corrected field size), computed distortion map, and an example of a deep UV image.
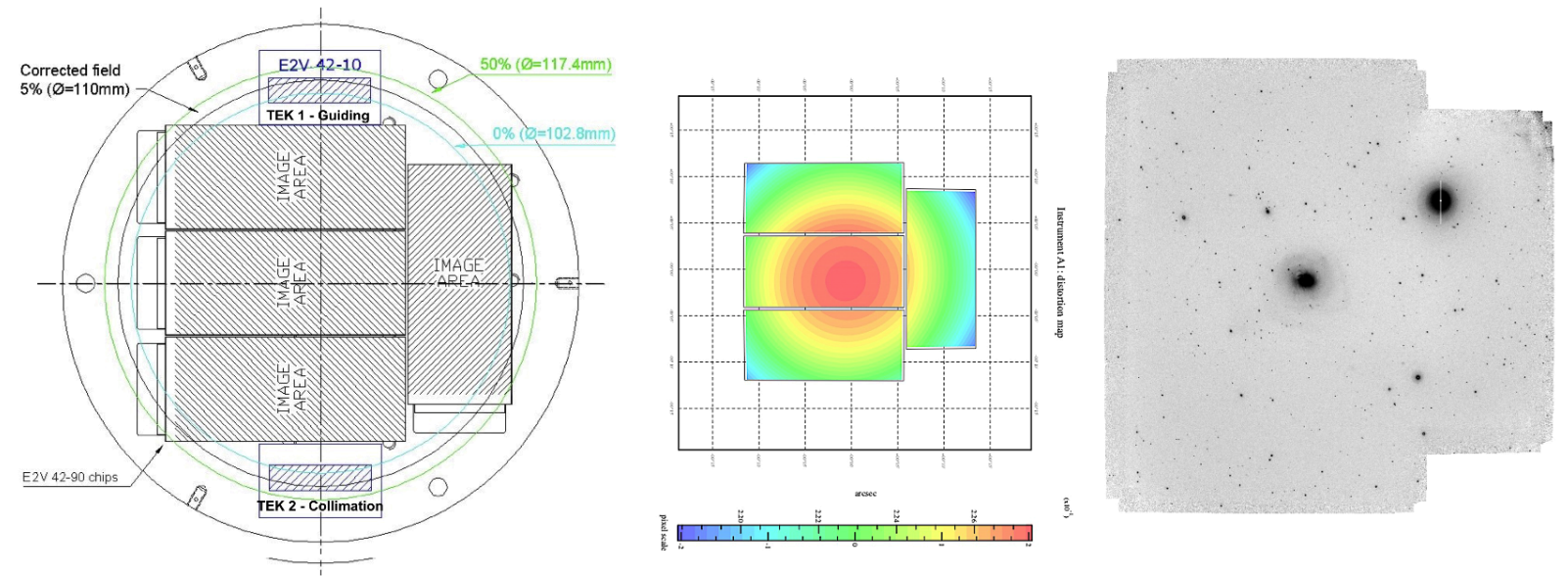

Figure 1: Shown here are: a) the chip layout of LBC Blue (science and technical chips); b) Distortion map computed for LBC Blue; and c) $2160 \mathrm{sec} U$-spec image of the galaxy merger. The project goal is to map the extent of the Globular Cluster (GC) and Young Star Cluster (YSC) population, which appear as partially or unresolved point sources across the field. Here, the LBC FOV covers $\sim 160 \times 150$ metric kiloparsecs, which should cover the entire spatial extent of the GC and YSC populations (Rochais et al ${ }^{22}$ ).

Each of the LBCs houses two filter wheels, each wheel houses 5 slots, which allows for up to 8 filters to be used for each instrument (one slot in each wheel must always be empty). Although nominally of similar design, the LBCs use different filters of different widths. LBC Blue filters are $159.8 \mathrm{~mm}$ in diameter (155 mm opening), while LBC Red filters are $189.6 \mathrm{~mm}$ in diameter (185 mm opening). Currently, LBC Blue houses six filters for scientific use: $U$-Bessel, $U$-Spectroscopic (broader transmission curve than $U$-Bessel), $B$-Bessel, $V$-Bessel, Sloan $g, r$. There are now ten filters available for scientific use with LBC Red: $V$-Bessel, $R$-Bessel, $I$-Bessel, sloan $r, i$, and $z$, and $Y$-band filter; and three medium filters, F972N20, TiO 784 and $\mathrm{CN} 817$. The TiO 784 and CN 817 filters were purchased and tested in semester 2014B by Landessternwarte Königstuhl (LBTB-Germany). These filters have been available for use by all LBTO partners since semester 2015A. However, they must be requested in advance to allow for time to swap with filters normally kept in the LBC Red filter wheels.

Steps continue toward the improvement of collimation with the LBCs. The goal is to expand and improve the range of conditions under which collimation can be achieved. Currently, collimation is achieved through a custom IDL program called DOFPIA which measures and analyzes the pupil (highly de-focused) images of stars, see Hill et al. $(2008)^{23}$ for more details. Using a geometrical method described by Wilson (1999) ${ }^{24}$ aberration coefficients are derived by measuring the internal and external borders of pupils, as well as in some cases, their illumination profiles. Empirically determined scaling relations based on these are then used to apply the Zernike corrections (Z4, Z5, Z6, Z6, Z8, Z11, and Z22) needed to remove the aberrations. A small region of Chip 2 is read-out in order to speed up the process. The process is repeated until the corrections converge.

In September 2015, during fall startup, the position of the region used for DOFPIA was changed to below 
Table 2. Overview of Available LBC Filters

\begin{tabular}{|c|c|c|c|c|c|}
\hline LBC Blue & $\begin{array}{l}\mathbf{5 0 \%} \text { Cut-On } \\
(\mu \mathrm{m})\end{array}$ & $\begin{array}{l}\mathbf{5 0} \% \text { Cut-Off } \\
(\mu \mathrm{m})\end{array}$ & LBC Red & $\begin{array}{l}\mathbf{5 0} \% \text { Cut-On } \\
(\mu \mathrm{m})\end{array}$ & $\begin{array}{l}\mathbf{5 0} \% \text { Cut-Off } \\
(\mu \mathrm{m})\end{array}$ \\
\hline$U$-Spectroscopic ${ }^{1}$ & 0.332 & 0.381 & $V$-Bessel & 0.493 & 0.577 \\
\hline$U$-Bessel & 0.333 & 0.382 & $r$-sloan & 0.553 & 0.686 \\
\hline$B$-Bessel & 0.375 & 0.469 & $R$-Bessel & 0.572 & 0.690 \\
\hline$g$-sloan & 0.397 & 0.550 & $i$-sloan & 0.697 & 0.836 \\
\hline$V$-Bessel & 0.488 & 0.610 & $I$-Bessel & 0.713 & 0.881 \\
\hline$r$-sloan & 0.552 & 0.686 & $z$-sloan & 0.830 & $\ldots$ \\
\hline$\ldots$ & $\ldots$ & $\ldots$ & $Y$ & 0.952 & 1.110 \\
\hline$\ldots$ & $\ldots$ & $\ldots$ & $\mathrm{TiO} 784^{2}$ & 0.769 & 0.788 \\
\hline$\ldots$ & $\ldots$ & $\ldots$ & $\mathrm{CN} 817^{2}$ & 0.802 & 0.821 \\
\hline$\ldots$ & $\ldots$ & $\ldots$ & $\mathrm{F} 972 \mathrm{~N} 20^{2}$ & 0.952 & 0.974 \\
\hline
\end{tabular}

${ }^{1}=$ Broad width (top-hat) filter response designed to mimic the spectroscopic coverage in this wavelength range; ${ }^{2}=$ Medium width filters

the rotator center on Chip 2 for both LBCs in an effort to improve collimation. The rotator center for LBC Blue is (in detector coordinates on Chip 2) $[1035,2924]$ and for LBC Red $[1078,2913]$. The new section is at $Y=1201: 2608$ (previous focus used $Y=3201: 4608$ ) and the entire width of Chip 2. DOFPIA needs to be run ever 30 minutes or less to maintain effective collimation. In addition, active collimation continues during science exposures when both technical chips takes exposures every 8-32 seconds (guiding and pupil exposures are set to the same exposure time, which is dependent on the brightness of the guidestar). Corrections from the collimation technical chip (Tek Chip 2) are applied in between science exposures. However, DOFPIA does have certain limitations that can affect achieving optimal collimation. This often occurs at the start of an observing night, where temperature differentials between the primary mirrors and ambient air make collimation time-consuming or difficult to achieve. The limitations, in part, come from issues in how DOFPIA fits the extrafocal pupils, as well as the signal-to-noise $(\mathrm{S} / \mathrm{N})$ of the data. A new system, Wavefront Reconstruction Software (WRS) is being developed with INAF. WRS takes into account $\mathrm{S} / \mathrm{N}$ considerations, applies a new wavefront reconstruction algorithm, and maintains a more detailed log of the corrections applied, (Stangalini et al. 2014 $4^{25}$ ). WRS testing indicates the biggest gains can be made when there is significant coma the start of the night (Z7 and/or Z8). WRS tests have been carried it during 2015 and 2016, but unfortunately have been hampered by poor weather during engineering nights. In some cases, WRS tests have been performed in parallel with science observations, mostly with LBC Red (DX) while MODS-1 or LUCI-1 is being used (SX). It is expected that a final analysis and a beta version will be made available for more widespread testing in the near future.

Improvements to the LBC control hardware and software continue. Software upgrades are regularly rolled out to correct and improve guiding, instrument rotation, non-sidereal guiding, and error handling. As noted in Summers et al. (2104) $\stackrel{26}{, 26}$ upgrades will be made to the LBC control systems since their installation at LBT. These include replacing the four CCD controllers, one each for LBC Blue and Red science CCDs and one each for Blue and Red guiding (technical controllers). Each controller is handled by a separate PC running Microsoft Windows Server 2003. An ethernet CCD controller upgrade will replace the need for four physical Windows PCs. Currently a single LINUX machine known as the Central Management Unit (CMU) is used to run the LBC software and interface with the CCD controllers. A future upgrade will eliminate the need for the Windows software and port everything to a LINUX based software system. A BeagleBones board ( \protect|http://beagleboard.org/) will be used to run LINUX on a daughter card attached to the CCD Controller cards. Step one (completed) is to move image analysis from the Windows PCs to the CMU. A Prototype CCD controller with a BeagleBones board is currently being tested by INAF, as is ongoing porting of software from Windows to LINUX. 


\subsection{Multi-Object Double Spectrograph (MODS)}

The Multi-Object Double Spectrographs (MODS) are a pair of identical optical imagers and spectrographs designed to use longslit and user-designed multi-object slit masks. Each MODS is attached to the straight through f/15 Gregorian focus on the respective primary mirror (MODS-1 on SX, MODS-2 on DX). MODS were designed and built by The Ohio State University as part of its contribution to the first generation of LBT instruments.

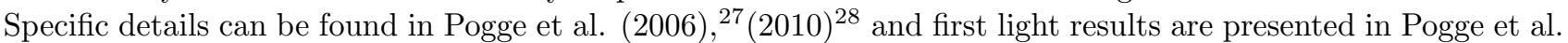
(2012) ${ }^{29}$ The instrument description and capabilities described applies to both MODS-1 and MODS-2. MODS1 was installed and aligned in 2009 and became available for partner science in semester 2011B. MODS-2 was installed in semester 2014A and commissioned in 2014B-2015. Both MODS have been used for on-sky science since semester 2015B and have been used in binocular mode in 2016.

MODS is a double spectrograph and imager that employs reflective optics to achieve high-throughput from near-UV $(0.32 \mu \mathrm{m})$ through near-IR $(1.1 \mu \mathrm{m})$ wavelengths. Both MODS house separate blue- and red-optimized channels that use custom-built E2V CCD231-68 back-side illuminated CCDs with $3072 \times 8192$ pixels $(15 \mu \mathrm{m}$ square). The blue channel is standard silicon with E2V Astro-Broadband coating and the red channel is $40 \mu \mathrm{m}$ thick deep depletion silicon with extended-red coating (E2V Astro-ER1). This provides increased performance long-wards of $0.8 \mu \mathrm{m}$, with significantly reduced fringing relative to other optical spectrographs and imagers. The readout time for the un-binned $8 \mathrm{~K} \times 3 \mathrm{~K}$ is $\sim 105$ seconds. For more information see Atwood et al. (2008) $\underline{30}$

The guiding and wavefront-sensing systems are constructed as part of MODS and located above the instrument focal plane, but within the unit itself. MODS also houses the calibration system internally. It consists of continuum (fixed intensity Quartz-Halogen and variable intensity incandescent) used for calibration imaging and spectroscopic flats; and emission-line lamps (arc lamps) used for wavelength calibration of grating and prism spectroscopy. The optical layout of MODS incorporates a dichroic beam splitter below the focal plane that splits light into separate, but optimized blue and red only channels. There is a cross-over at $0.565 \mu \mathrm{m}$ that results in a drop in flux in a small region $(\sim 0.005 \mu \mathrm{m}$ centered on this wavelength). For some science cases, users may choose to employ blue- or red-only observations. The dichroic is replaced with no optic in the beam for blue-only mode and replaced with a flat mirror for red-only mode (imaging and spectroscopy). MODS uses an infrared laser $(\lambda=1.55 \mu \mathrm{m})$ closed-loop image compensation system (IMCS) to provide flexure compensation due to gravity, mechanical, and temperature effects. The IMCS can null motion to within an average of \pm 0.6 pixels for every $15^{\circ}$ for elevations of $90^{\circ}-30^{\circ}$. More information about the IMCS can be found in Marshall et al. (2006)! $\underline{31}$

MODS has two observing modes: direct imaging, and spectroscopy using curved focal plane masks. These masks include facility longslit and multi-object slit masks that can be custom designed by users and fabricated at the University Research Instrumentation Center (URIC) at the University of Arizona (see Reynolds et al. $2014^{32}$ for details on fabrication and materials used for the masks). Direct imaging is achieved by replacing the grating with a plane mirror and is used for target acquisition for spectroscopy. The standard acquisition is to read out a smaller $1 \mathrm{~K} \times 1 \mathrm{~K}$ region of the $\mathrm{CCD}$ to reduce overheads during the acquisition (readout $\sim 40 \mathrm{sec}$ ). Direct imaging can also be used for science programs. MODS includes a full complement of sloan filters: $u$, and $g$ for the Blue channel; and $r, i$, and $z$ for the Red channel. The usable FOV is $6^{\prime} \times 6^{\prime}$ but with degraded image quality at radii $>4^{\prime}$. In the case of direct imaging for science, the CCDs are read out in $3 \mathrm{~K} \times 3 \mathrm{~K}$ mode (readout time is $\sim 68 \mathrm{sec}$ ).

MODS has two spectroscopic modes: a medium resolution diffraction grating optimized for blue and red spectral regions with $R \sim 2300$, and 1850 (using a $0^{\prime \prime} .6$ wide slit, and scaling inversely with increasing slitwidth), respectively; and a double-pass $8^{\circ}$ glass prism with back reflective coating that produces a low-dispersion spectroscopic mode with $R \sim 420-140$ in the blue, and $R \sim 500-200$ in the red. The grating dispersion uses the full $8 \mathrm{~K} \times 3 \mathrm{~K} \mathrm{CCD}$, while the prism mode uses a $4 \mathrm{k} \times 3 \mathrm{~K}$ readout mode. Longslit and multi-object slit masks are made available through a mask cassette system with 24 positions. Each mask is matched to the shape of the Gregorian focal plane. The first 12 positions in the cassette contain permanent facility and testing masks. The facility science masks include: $0^{\prime \prime} .3,0^{\prime \prime} .6,0^{\prime \prime} .8,1^{\prime \prime} .0$, and $1^{\prime \prime} .2$ longslit segmented masks (each contains five $1^{\prime}$ long slits each separated by segmented braces); and a $5^{\prime \prime}$ wide $\times 60^{\prime \prime}$ long longslit single segment mask used primarily for spectro-photometric calibrations. In semester $2016 \mathrm{~A}$ a new facility $2^{\prime \prime} .4 \times$ segmented longslit was fabricated and is now available for all LBT partners upon request. The remaining 12 mask slots are available for custom designed MOS masks (discussed in the next section).

MODS has excellent sensitivity at both the UV and near-IR extremes, producing high S/N spectra as far 
blue-ward as $0.315 \mu \mathrm{m}$ and as far red-ward as $1.05 \mu \mathrm{m}$. Table 3 provides an overview of the imaging and spectroscopic modes available for MODS-1 and MODS-2.

Table 3. Overview of MODS-1/-2 Configurations

\begin{tabular}{|l|l|l|l|l|l|}
\hline Mode & Channel & Filters & $\begin{array}{l}\text { Resolution } \\
\left(0^{\prime \prime} .6 \text { slit }\right)\end{array}$ & $\begin{array}{l}\lambda \\
(\mu \mathrm{m})\end{array}$ & CCD Size \\
\hline Imaging & Dual & $u, g, r, i, z$ & $\ldots$ & $0.33-0.95$ & $3 \mathrm{~K} \times 3 \mathrm{~K}$ \\
& Blue & $u, g$ & $\ldots$ & $0.33-0.55$ & \\
& Red & $r, i, z$ & $\ldots$ & $0.55-0.95$ & \\
\hline Grating & Dual & Clear & $1850-2300$ & $0.31-1.05$ & $8 \mathrm{~K} \times 3 \mathrm{~K}$ \\
Spectroscopy & Blue & Clear & $1850(@ 0.4 \mu \mathrm{m})$ & $0.32-0.60$ & \\
& Red & GG495 & $2300(@ 0.7 \mu \mathrm{m})$ & $0.50-1.05$ & \\
\hline Prism & Dual & Clear & $500-140$ & $0.31-1.05$ & $4 \mathrm{~K} \times 3 \mathrm{~K}$ \\
Spectroscopy & Blue & Clear & $420-140$ & $0.32-0.60$ & \\
& Red & GG495 & $500-200$ & $0.50-1.05$ & \\
\hline
\end{tabular}

Figure 2 shows an example spectrum obtained with MODS-1, using the dual-grating mode with high sensitivity at both near-UV and near-IR wavelengths. The target is a z 1 Ultraluminous Infrared Galaxy (ULIRG) that is suspected of being a late-stage merger between two gas-rich spiral galaxies (Rothberg et al. 2015 53 ). ULIRGs emit $10^{12} L_{\odot}$ integrated over 8-100 $\mu \mathrm{m}$ and contain anywhere from $10^{9}-10^{10} M_{\odot}$ of molecular gas, which provides fuel for forming new stars and growing super-massive central black holes (SMBH) that power Active Galactic Nuclei (AGN). The most powerful AGN are quasars (QSOs) and reside in massive elliptical galaxies (10-100x more massive than the Milky Way). In the local Universe, ULIRGs are known as the progenitors of QSO host galaxies (e.g. Sanders et al. 1988, $\frac{34}{34}$ Rothberg et al. 2013

\subsubsection{MODS Multi-Object Spectroscopic (MOS) Masks}

The multi-object spectroscopy (MOS) masks allow PIs to create masks based on the scientific needs of the targets to be observed. Masks are designed using a software program called MMS (MODS Mask Simulator). The software is a modified version of the LUCI mask software, LMS (LUCI Mask Simulator). A user's manual can be found at www.astronomy.ohio-state.edu/ martini/mms/. Both are based upon the European Southern Observatory SkyCat tool. The software allows users to load a fits image file with a valid world coordinate system (WCS) or access archival images from the Digital Sky Survey or 2MASS (2 Micron All Sky Survey) and place slits of userdefined length and width within the field of view of MODS. The MMS software allows users to rotate the image as needed, add multiple slits, and display information to ensure that slits do not overlap. Alignment is done using a minimum of three $4^{\prime \prime} \times 4^{\prime \prime}$ alignment boxes that are placed over the positions of stars in the field. Smaller sized boxes may be used if needed, but they should be larger than the upper limits of the required seeing constraints (so the star may be fully measured in the box). The MODS alignment software (modsAlign) uses these boxes to determine offset and rotation to align the mask with the target field. The newest version of modsAlign used for both MODS auto-detects the alignment boxes using the measured focal-plane to detector geometries and then prompts users to centroid on the alignment stars that should be centered within the box. The more stars used, the more precise the alignment. The software compares the centroid position of the stars and the positions of the alignment boxes to determine the offsets in translation and rotation. The MMS software also requires users to select a valid guidestar, and provides an overlay of the Auto-Guiding and Wavefront sensing (AGW) patrol field. Figure 3 shows the MMS software with an example target and MOS mask being created (left) and an example of the final mask output as Gerber $(g b r)$ file. Masks are submitted by the partner coordinators to the LBTO Mask Scientist at various mask deadlines during each semester. The MOS scientist (currently, B. Rothberg) reviews each mask to ensure it meets the criteria of sufficient alignment boxes, no overlapping slits, a suitable guidestar, etc. Approved masks are then sent to URIC for fabrication. For more information on fabrication and materials 


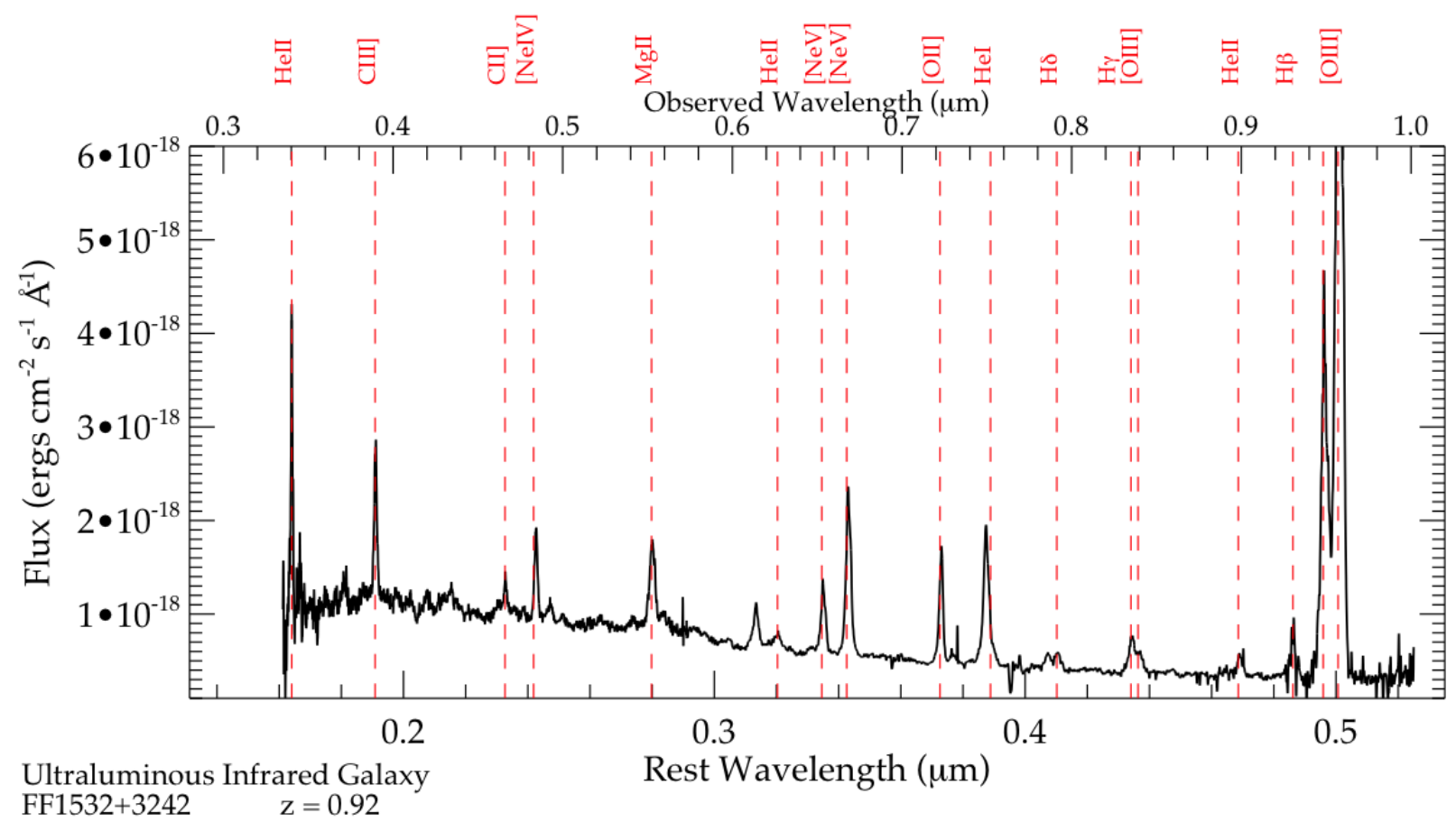

Figure 2: Shown is a sample spectrum from a project to measure the dynamical, star-formation, and search for AGN in a sample of Ultraluminous Infrared Galaxy mergers at $0.4<\mathrm{z}<1.0$. The sample selection is different than most intermediate redshift ULIRG surveys (PI Rothberg). Rest-frame UV/Optical spectra were obtained from MODS-1/-2 in and used in conjunction with archival imaging from Hubble Space Telescope. The spectra shown here were obtained with MODS-1, $4800 \mathrm{sec}$ total integration time, $0^{\prime \prime} .6 \mathrm{slit}$, and dual grating mode. Both the observed and rest-frame wavelengths are shown to demonstrate the range of MODS and identify the highexcitation emission lines. The $\mathrm{z}=0.92$ ULIRG shows evidence of a SMBH with $M_{\bullet} \sim 10^{8} M_{\odot}$, consistent with the SMBH masses in nearby Type I AGN, like Mrk 231.

used, (Reynolds et al. 2014 ${ }^{32}$ ). Once fabricated they are sent to LBTO and mask IDs are used to catalog the masks and place them into inventory for future (and possibly repeated) use. Mask exchanges are typically done just before or during the day of the first partner science block. Unlike LUCI, the mask unit and masks are not cryogenically cooled, allowing each partner science block to use all 12 of the available mask slots. Multiple mask exchanges can be done fairly quickly during partner science blocks if more than 12 MOS masks are needed.

\subsubsection{Binocular Observing with MODS-1 \& MODS-2}

The use of MODS-1 \& MODS-2 together for scientific observations marks the second of the three facility instruments ready for binocular operations on-sky. The first tests of the binocular mode of MODS-1 \& MODS-2 took place on UT January 15, 2016. The instrument PI, Richard Pogge (Ohio State University) developed an interface that takes a single MODS script and "twins" it so that the preset (or pointing information) instructs the binocular mount to move to a designated set of celestial coordinates and configures both mirrors to point at the same region of the sky. The observers simply run a shell script, for acquisition (acqBinoMODS) or for starting science observations (execBinoMODS) which automatically twins the single input script. For spectroscopy, after the preset, the observers must then align and place the science target in the longslit for each MODS separately. In the case of imaging, observers execute a shell script that moves the telescope to the science field and begins the science integrations on both MODS.

Figure 4 shows the first dual grating spectra ( $1^{\prime \prime} .0$ slitwidth) obtained simultaneously from MODS-1 and MODS-2 of the nearby Seyfert 2 AGN host galaxy NGC 1068. The data were processed using a quick-look 


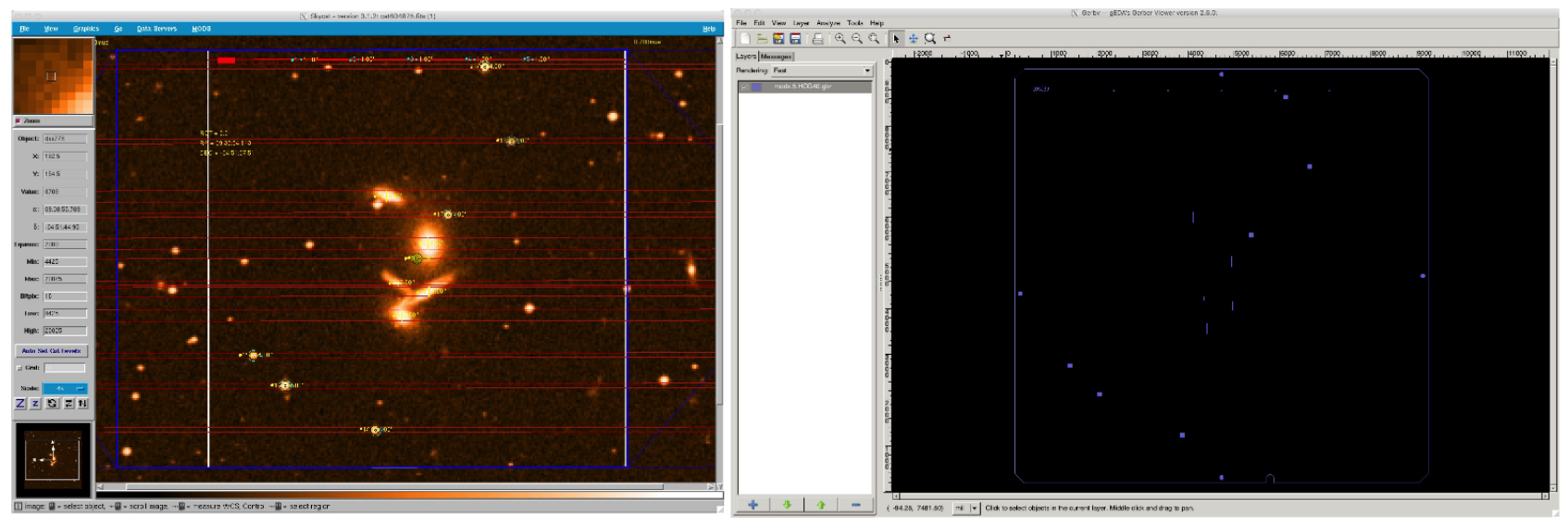

Figure 3: MODS MMS software used to design masks MOS masks (left). The example shown uses Hickson Compact Group 40, a group of five galaxies gravitationally bound to each other, several of which are in the early stages of interaction. MMS creates a Gerber file $(g b r)$ which has the information needed to physically manufacture the mask. Note the square reference boxes used for alignment, and science slits $\left(0^{\prime \prime} .6\right.$ width and of $4^{\prime \prime}-10^{\prime \prime}$ in length).

software developed by The Ohio State University (and based upon the modsIDL data reduction package: \protecthttp://www.astronomy.ohio-state.edu/MODS/Software/modsIDL/) and currently available for visiting astronomers to use to assess the quality of data obtained in near real time. A total of three targets, including an intermediate redshift ULIRG, were successfully observed that night using the MODS-Binocular mode. The MODS-Binocular mode has been available on a "shared-risk" basis for visiting astronomers since May 2016, allowing all LBT partners an opportunity to use both MODS. As of semester 2016A, only longslit-longslit or imaging-imaging configurations are supported. MOS masks are not currently supported for MODS-Binocular mode, but should be available for use in semester 2016B. This will require two masks to be fabricated from a single $g b r$ file. The next step is to use mixed configurations, such as different MOS masks for the same field, or a mixture of spectroscopy and imaging. Nevertheless, MODS-Binocular currently provides PIs a $\sqrt{2}$ increase in $\mathrm{S} / \mathrm{N}$ for the same amount of observing time as before.

\subsection{LBT NIR Spectroscopic Utility with Camera Instruments (LUCI)}

The two LBT Utility Camera in the Infrared instruments (LUCI, formerly LUCIFER), are a pair of cryogenic near-IR (NIR) instruments, capable of imaging and spectroscopy (longslit and MOS) each located at a bent Gregorian $f / 15$ focus port of the SX and DX mirrors. The discussion of the LUCIs will focus primarily on seeing-limited operations. Diffraction-limited modes for both LUCIs are still being commissioned. The LUCIs are rather compact and rely on a series of fold mirrors to bring the light from the tertiary mirror (M3) into the focal plane. The LUCIs are cooled using closed cycle coolers which are monitored to maintain the correct temperatures needed for optimal operation. Currently, flexure compensation is achieved in a passive mode whereby a lookup-table is used based on the elevation and rotation of the instrument and applied before an exposure is taken. The corrections are applied to the last fold mirror in the optical train (FM4), which lies in front of the instrument's internal pupil. An active flexure compensation system is currently in development that will apply corrections during a science exposure.

The LUCIs are sensitive from 0.95-2.44 $\mu \mathrm{m}$ and are designed to be used in both seeing-limited and diffraction limited (via active optics) modes. More detailed technical information and on-sky performance about LUCI

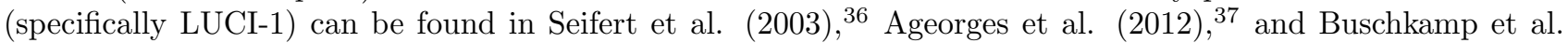
(2012). $\frac{38}{6 U C I-1}$ was installed at LBT in September 2008 and has been in service from December 2009 through July 2015 in seeing-limited mode only. It was removed from the telescope during 2015 summer shutdown to replace the detector with a Hawaii2 $\mathrm{RG}(\mathrm{H} 2 \mathrm{RG}) 2 \mathrm{~K} \times 2 \mathrm{~K}$ detector and install a high resolution camera (N30) which is designed to be used with adaptive optics (AO). These upgrades were designed so LUCI-1 would match 


\section{MODS1+2 Binocular First Light - 2016 Jan 15}

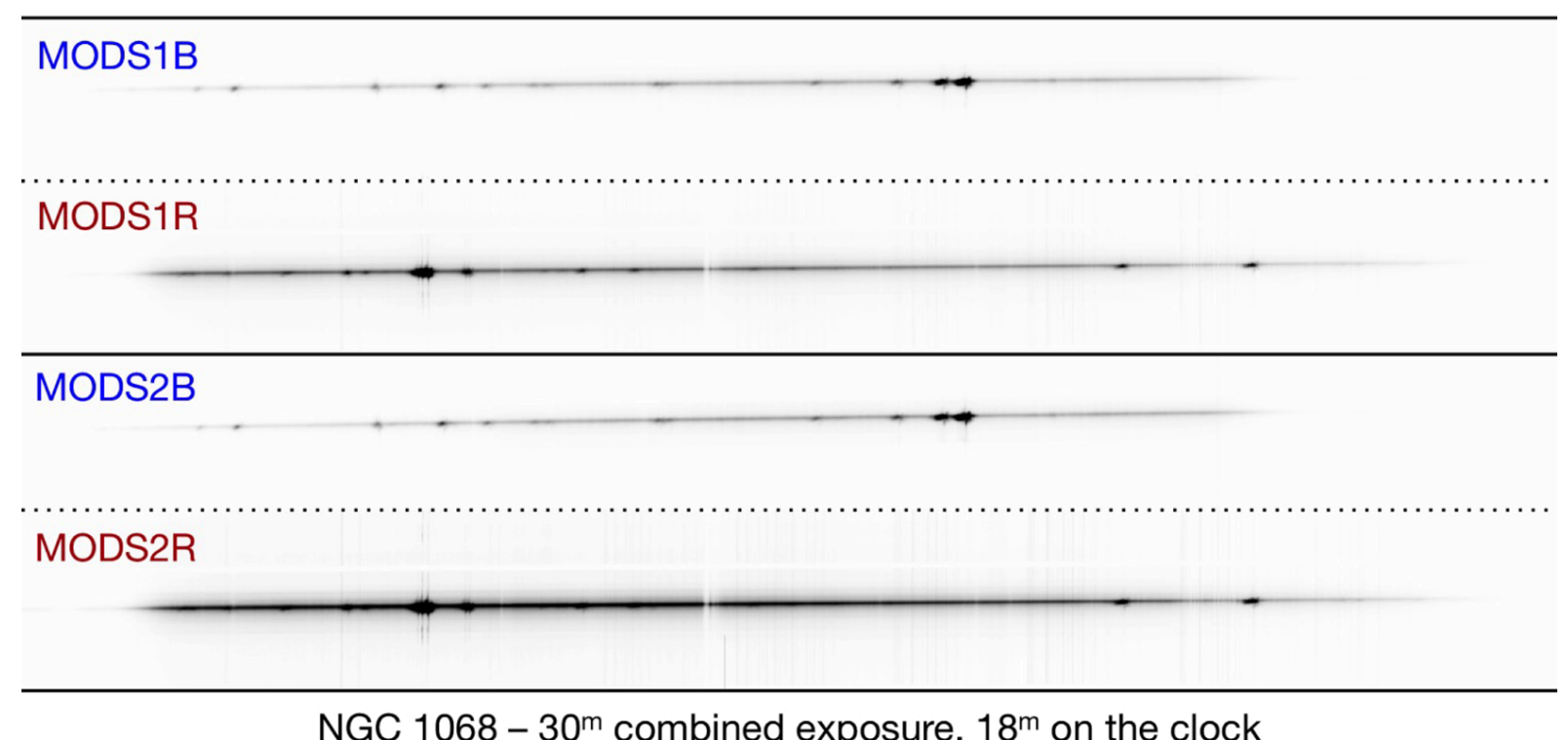

NGC $1068-30^{m}$ combined exposure, $18^{m}$ on the clock

Figure 4: First MODS-Binocular observations of a single target, the Seyfert 2 AGN NGC 1068. Both MODS were configured in dual grating mode with a $1^{\prime \prime} .0$ slitwidth. The total exposure time was 15 minutes per channel and mirror (300 sec $\times 3$ exposures), or 30 minutes total each for blue and red channels. Thus, including overheads, 30 minutes worth of data were obtained in $\sim 18$ minutes. The initial binocular acquisition preset was sent by the instrument PI Richard Pogge. The MODS-Binocular observations that night were executed from the remote observing room in Tucson.

the capabilities of LUCI-2. LUCI-2 was made available to the LBT community for on-sky science starting in semester 2015B and continuing through semester 2016A in seeing-limited mode only. Commissioning of the diffraction-limited modes of LUCI-1 and LUCI-2 are ongoing. In addition, both LUCIs are designed to work with the ARGOS, a green laser system designed for wide-field ground-layer adaptive optics (GLAO) corrections, (e.g. Rabien et al. 2010, ${ }^{39}$ Rabien et al. 2014, 40 and Rahmer et al. 2014는 $)$.

Both LUCI-1 and LUCI-2 are now equipped with the same $2 \mathrm{~K} \times 2 \mathrm{~K}$ H2RG detectors. The detectors are controlled by GEIRS (GEneric InfraRed detector Software) developed by MPIA. The LUCIs now have the same set of cameras: an $f / 1.8$ camera with $0^{\prime \prime} .25$ pixel $^{-1}(\mathrm{~N} 1.8)$, an $f / 3.75$ camera with a $0^{\prime \prime} .12$ pixel $^{-1}$, and an $f / 30$ camera with $0^{\prime \prime} .015$ pixel $^{-1}$. Nominally, the N1.8 camera is primarily used for seeing-limited spectroscopy; the N3.75 camera is used for seeing-limited imaging, yielding a $4^{\prime} \times 4^{\prime} \mathrm{FOV}$; and the N30 is used for AO imaging and spectroscopy, providing a $30^{\prime \prime} \times 30^{\prime \prime}$ FOV. Both LUCIs also house the same complement of broad and narrow-band filters. However, there are differences between the available spectroscopic gratings for the two LUCIs. Tables 4 \& 5 provide an overview of the capabilities available for both LUCIs in seeing-limited mode. Unlike MODS, where the grating tilt is not changeable by the user, the LUCIs offer a wide range of configuration possibilities that can be achieved with various tilts (i.e. central wavelengths or $\lambda_{\mathrm{c}}$ ), gratings, slits, and cameras. Using the N1.8 camera, low resolution grating (G200) permits nearly complete coverage of the near-IR window with only two settings. The high resolution grating (G210) with the N1.8 camera allows for nearly full wavelength coverage of each filter (i.e. $z, J, H$, and $K$-band). Users also have the flexibility to combine cameras, gratings, slits, and $\lambda_{\mathrm{c}}$ in different ways to achieve a wide range of scientific goals (i.e. higher spectral resolutions over shorter wavelength ranges).

The calibration unit for both LUCIs are external the instrument. The units are mounted above the bent Gregorian ports housing LUCI. In stowed position, they are flush with the ports. When required, they are 
Table 4. LUCI-1 \& LUCI-2 Filters Available for Science

\begin{tabular}{|c|c|c|c|c|c|}
\hline Filter & $\begin{array}{l}\lambda_{C} \\
(\mu \mathrm{m})\end{array}$ & $\begin{array}{l}\text { FWHM } \\
(\mu \mathrm{m})\end{array}$ & Filter & $\begin{array}{l}\lambda_{\mathrm{c}} \\
(\mu \mathrm{m})\end{array}$ & $\begin{array}{l}\text { FWHM } \\
(\mu \mathrm{m})\end{array}$ \\
\hline$z$ & 0.957 & 0.195 & He I & 1.088 & 0.015 \\
\hline$J$ & 1.247 & 0.305 & Paschen- $\gamma$ & 1.097 & 0.010 \\
\hline$H$ & 1.653 & 0.301 & OH 1190 & 1.194 & 0.010 \\
\hline$K_{\mathrm{s}}$ & 2.163 & 0.270 & $J$ low & 1.199 & 0.112 \\
\hline$K$ & 2.104 & 0.408 & Paschen- $\beta$ & 1.283 & 0.012 \\
\hline$z J$ spec & 1.175 & 0.405 & $J$ high & 1.303 & 0.108 \\
\hline$H K$ spec & 1.950 & 0.981 & FeII & 1.646 & 0.018 \\
\hline$Y 1$ & 1.007 & 0.069 & $\mathrm{H}_{2}$ & 2.124 & 0.023 \\
\hline $\mathrm{OH} 1060$ & 1.065 & 0.010 & Brackett- $\gamma$ & 2.170 & 0.024 \\
\hline$Y 2$ & 1.074 & 0.065 & $\ldots$ & $\ldots$ & $\ldots$ \\
\hline
\end{tabular}

Table 5. LUCI-1 \& LUCI-2 Orders, Gratings, Valid $\lambda_{\mathrm{c}}$, and Resolution Values for Seeing-Limited Mode

\begin{tabular}{|c|c|c|c|c|c|c|}
\hline Order & $\begin{array}{l}\text { G210 HiRes } \\
\text { Valid } \lambda_{\mathrm{c}}(\mu \mathrm{m}) \\
\end{array}$ & $\begin{array}{l}\Delta \lambda \\
(\mu \mathrm{m})\end{array}$ & $\begin{array}{l}\text { Resolution } \\
\left(0^{\prime \prime} .5 \text { slit }\right) \\
\end{array}$ & $\begin{array}{l}\text { G200 LoRes } \\
\text { Valid } \lambda_{\mathrm{c}}(\mu \mathrm{m}) \\
\end{array}$ & $\begin{array}{l}\Delta \lambda \\
(\mu \mathrm{m})\end{array}$ & $\begin{array}{l}\text { Resolution } \\
\left(0^{\prime \prime} .5 \text { slit }\right) \\
\end{array}$ \\
\hline 1 & $\begin{array}{l}\cdots \\
\ldots\end{array}$ & $\begin{array}{l}\cdots \\
\ldots\end{array}$ & $\begin{array}{l}\cdots \\
\ldots\end{array}$ & $\begin{array}{l}1.476-2.535 \text { (L1) } \\
1.248-2.491 \text { (L2) }\end{array}$ & $\begin{array}{l}0.880 \\
"\end{array}$ & $\begin{array}{l}1900(\mathrm{H}), 2600(\mathrm{~K}) \\
"\end{array}$ \\
\hline 2 & $\begin{array}{l}2.098-2.728(\mathrm{~L} 1) \\
2.061-2.702(\mathrm{~L} 2) \\
\end{array}$ & $\begin{array}{l}0.328 \\
"\end{array}$ & $\begin{array}{l}5000 \\
"\end{array}$ & $\begin{array}{l}0.897-1.358^{*}(\mathrm{~L} 1) \\
0.599-1.246^{*} \text { (L2) }\end{array}$ & $\begin{array}{l}0.440 \\
"\end{array}$ & $\begin{array}{l}2100(\mathrm{z}), 2400(\mathrm{~J}) \\
"\end{array}$ \\
\hline 3 & $\begin{array}{l}1.398-1.819 \text { (L1) } \\
1.374-1.801 \text { (L2) }\end{array}$ & $\begin{array}{l}0.202 \\
"\end{array}$ & $\begin{array}{l}5900 \\
"\end{array}$ & $\begin{array}{l}\cdots \\
\cdots\end{array}$ & $\begin{array}{l}\cdots \\
\cdots\end{array}$ & $\begin{array}{l}\cdots \\
\cdots\end{array}$ \\
\hline 4 & $\begin{array}{l}1.084-1.364(\mathrm{~L} 1) \\
1.072-1.351(\mathrm{~L} 2)\end{array}$ & $\begin{array}{l}0.150 \\
"\end{array}$ & $\begin{array}{l}5800 \\
"\end{array}$ & $\begin{array}{l}\cdots \\
\cdots\end{array}$ & $\begin{array}{l}\cdots \\
\cdots\end{array}$ & $\begin{array}{l}\cdots \\
\cdots\end{array}$ \\
\hline 5 & $\begin{array}{l}0.839-1.075^{*} \text { (L1) } \\
0.824-1.066^{*} \text { (L2) }\end{array}$ & $\begin{array}{l}0.124 \\
"\end{array}$ & $\begin{array}{l}5400 \\
"\end{array}$ & $\begin{array}{l}\cdots \\
\cdots \\
\cdots\end{array}$ & $\begin{array}{l}\cdots \\
\cdots \\
\cdots\end{array}$ & $\begin{array}{l}\cdots \\
\cdots \\
\cdots\end{array}$ \\
\hline
\end{tabular}

L1 is LUCI-1, L2 is LUCI-2. ${ }^{*}=$ While $\lambda_{\mathrm{c}}<0.95 \mu \mathrm{m}$ are valid, the L1 and L2 entrance windows are now coated to cutoff at $\lambda<0.95 \mu \mathrm{m}$. Resolution scales down as slitwidth increases. The $z J$ spec and $H K$ spec filters in Table 4 are primarily used with the G200 grating. The G150 Ks grating is only available on LUCI-1 and the allowable $\lambda_{\mathrm{c}}$ wavelength range is 1.95-2.4 $\mu \mathrm{m}$ and $\Delta \lambda=0.533 \mu \mathrm{m}$ with $R \sim 4150$ using a 2 pixel slitwidth $\left(0^{\prime \prime} .5\right)$. The G040 AO grating is only available on LUCI-2. More information regarding the G040 AO grating will be determined at a later date. The wavelength coverage in this table assumes the N1.80 camera. If using the N3.75 camera multiply $\Delta \lambda$ by 0.48 , and if using the N30 camera multiple by 0.06 . This table should allow one to determine the wavelength range visible for different configurations.

activated by scripts (or manually from the user interface or WEB/IO interface) and swing out laterally and then downwards so they are directly in front of the entrance window. Three Halogen lamps of varying brightness are used for imaging and spectroscopic flats. In 2016A, a neutral density filter was added to the LUCI-2 calibration unit to better match the intensities of the LUCI-1 calibration unit. Three emission-lamps (arc lamps) are available for wavelength calibration: Neon, Argon, and Xenon. 


\subsubsection{LUCI Multi-Object Spectroscopic (MOS) Masks}

LUCI-1 and LUCI-2 each use a cryogenic MOS unit to house both a set of permanent facility longslit masks and user designed MOS slit masks (Hofmann et al. 2004를 and Buschkamp et al. 2010 43 ). The MOS units hold up to 33 masks distributed over two cabinets. The permanent cabinets, which are inside the LUCIs, houses 10 facility masks, including longslit masks. These include: a wide mask with two long slits, one $2^{\prime \prime}$ wide (top) and one $1^{\prime \prime} .5$ wide (bottom), both $100^{\prime \prime}$ in length; and longslits of length $3^{\prime} .8$ and widths of $1^{\prime \prime} .0,0^{\prime \prime} .75$ (currently only available in LUCI-2), $0^{\prime \prime} .5,0^{\prime \prime} .25$, and $0^{\prime \prime} .13$ (to be used with AO and the N30 camera). This main unit houses the focal plane unit (FPU) which places the masks in and out of the LUCI focal plane using a robotic grabber arm (see Figure 5). The grabber slides along set of rails to select the requested mask, place it in the FPU, and later place the mask back in its designated slot once it is no longer needed (and another mask is requested). When imaging mode is used, an empty mask holder is placed in the FPU to allow light to pass unobstructed to the detector. A second exchangeable cassette which contains 23 masks slots is used to house MOS masks custom designed by science PIs. Secondary cabinet exchanges are executed on a monthly basis to accomodate different partner science programs. The exchanges include masks from multiple partners, with each partner assigned a maximum number available slots in the secondary cabinet. Mask exchanges are performed at cryogenic temperatures and require the use of two auxiliary cryostats in order to maintain pressure and temperatures at all times. An auxiliary cryostat holding a secondary cabinet is loaded with the next set of masks to be used for science. It is evacuated and cooled over 24-48 hours before a scheduled exchange. During the exchange, one aux cryostat is attached to LUCI using a set of gate valves controlled by software. Rails connect the aux cryostat to LUCI. The current installed secondary cabinet is moved along the rails into the cryostat. That cryostat is removed and a second cryostat is then attached and a secondary cabinet containing the new masks is placed into LUCI. The cabinet exchange is all done on the telescope infrastructure itself. This requires the cryostats to be lifted up through large doors in the high bay up and over the telescope and then gently placed on a platform on the telescope (located between the SX and DX mirrors where the bent Gregorian foci are located)

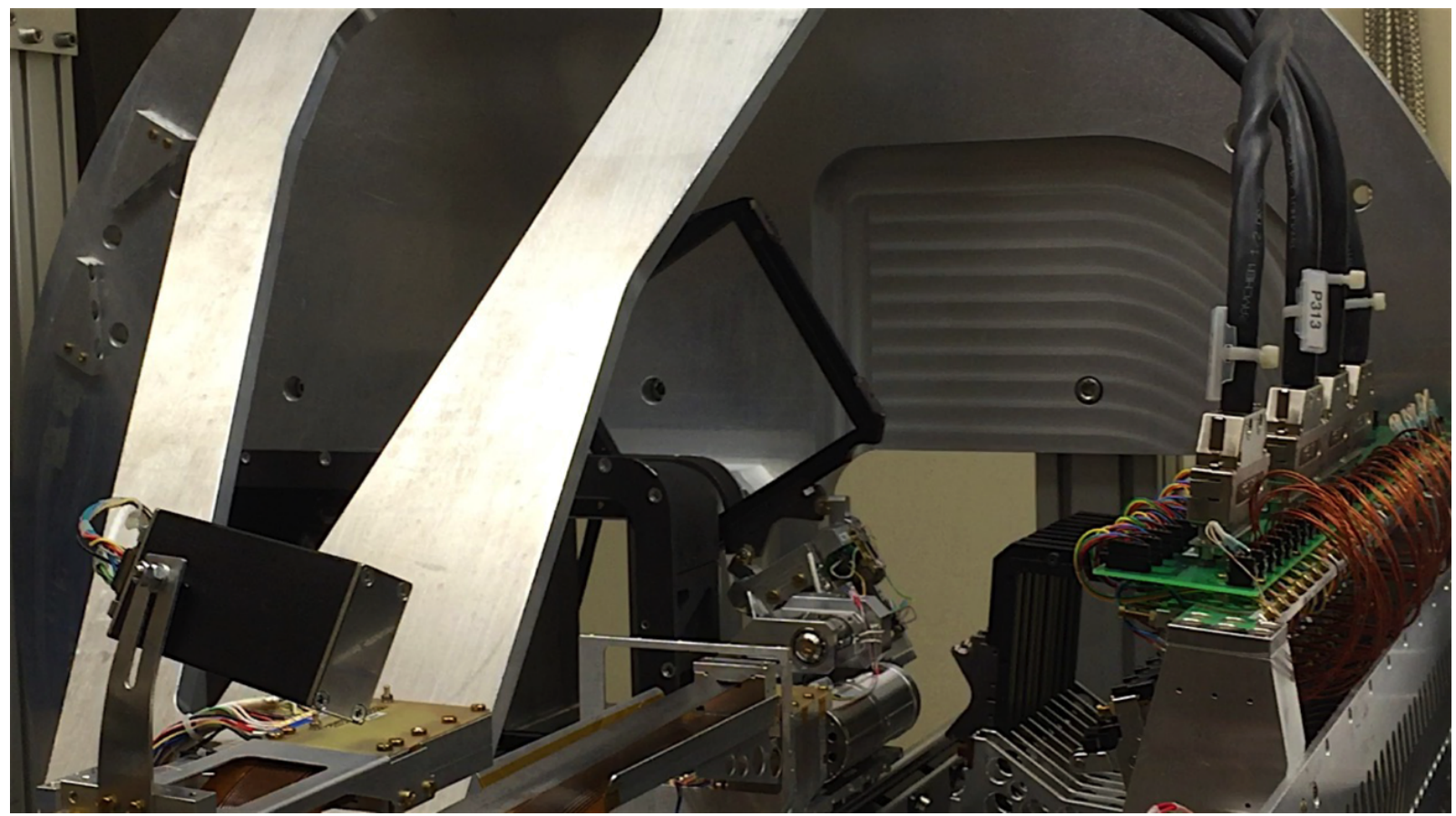

Figure 5: The LUCI-1 MOS unit outside of its housing and only with the permanent cabinet. Shown is the grabber arm placing a mask holder (which would normally contain a MOS or longslit mask) into the FPU. The rail system can be seen at the bottom of the image along with the slots holding other masks in place (right side of photo). Photo courtesy of B. Rothberg. 
The LMS software is used to create MOS mask designs (\protecthttp://abell.as.arizona.edu/ lbtsci/Instruments/LUCIFER/ It is the forerunner of MMS. The interface and concept is nearly the same as MMS (see Figure 3). The main differences are the the different guidestar patrol fields (generally above the science FOV for LUCI and generally below the science FOV for MODS), and that LUCI uses reference stars to calculate the rotation and shift needed to align the mask correctly. A previous version of the LUCI control software only required alignment stars to be defined by LMS without the needs for alignment boxes to be cut in the mask around each star. Currently, the new version of the LUCI software requires both designated reference stars and alignment boxes cut around them, but future updates may return to a system that does not require physical alignment boxes cut into the mask. MOS mask designs are submitted at the same deadline as MODS MOS mask designs. As with MODS, the MOS scientist reviews each mask to ensure it meets the criteria of sufficient alignment boxes, no overlapping slits, a suitable guidestar, etc. Approved masks are then sent to URIC for fabrication. For more information on fabrication and materials used, see Reynolds et al. 2014. ${ }^{32}$ Once fabricated they are sent to LBTO and mask IDs are used to catalog the masks and place them into inventory for future (and possibly repeated) use.

\subsubsection{LUCI Software Upgrade}

As noted above, a series of hardware upgrades were made to LUCI-1 during summer shutdown of 2015 . In addition to hardware upgrades, a new LUCI User interface has been developed by MPIA. The software completely replaces the previous version used to run LUCI-1. The new LUCI User interface communicates directly with both LUCI-1 and LUCI-2, and in principle, allows for binocular control of both instruments. The interface has three major components (see Figures 6 and 7): 1) The Main Observer Graphical User Interface (GUI) which displays information such as target coordinates, name, offsets, instrument configuration, and integration times in the central "queue" panel. Users load the script and click "GO" which sends the preset information to the telescope control software (TCS) and configures the instrument. Users may Pause, Reset, Abort, and Skip (or skip to) steps; 2) the Real-Time Display (RTD), which is based on the ALADIN software (\protect/http://aladin.ustrasbg.fr/) and is used for longslit and MOS mask acquisition; and 3) the Readout and Instrument Control panels, which gives users the ability to manually change parameters such as filters, mask, integration times, cameras, and the calibration unit. The previous LUCI-1 software parsed scripts using ASCII plain text. The new LUCI control software requires observing scripts in XML format. Currently, a scripting webpage, SCRIPTOR (\protect|http://scriptor.tucson.lbto.org/), was created to allow users to generate XML scripts by selecting the instrument, its configuration, integration times, guidestars, etc.

\section{MIXED-MODE USE}

The goal of LBT is to use the telescope in binocular mode all of the time. LBTI and LBCs have been observing in binocular mode for some time. In the last few months, MODS have been successfully tested and used on-sky in binocular mode. While the facility instruments have been designed to work in pairs in binocular mode, the telescope can also be figured to use instruments in a "mixed mode." These modes would see configurations such as MODS-1/LBC-R, MODS-2/LBC-B, LUCI-1/LBC-R, LUCI-2/LBC-B, or MODS/LUCI. Mixed-Mode use is desirable as it opens up a much wider wavelength ranges for scientific study (i.e. simultaneous UV and near-IR observations). As noted in Hill et al. (2014), $\frac{44}{4}$ the two sides are not required to have precisely the same target or position angle for binocular mode to work. The telescope mount points near the the mid-point between the two sides and the telescope software "knows" to avoid presets or small offsets that would violate the co-pointing limit (the maximum travel distance between the two mirrors). Currently, the co-pointing limit is set by software to be $40^{\prime \prime}$ (radius) apart in any direction. In effect, once the telescope mount has slewed to a set of coordinates, the two mirrors can effectively be treated as independent telescopes. Each side can dither as required by the science, so long as the two sides together don't violate the co-pointing limit.

However, a current limitation of using Mixed-Mode is the ability to pass a binocular preset from two different instruments to the TCS. Since 2014, several combinations of Mixed-Mode have been used. Currently, different combinations require somewhat different setups and have different limitations. The first attempts in 2013 used a LUCI-1/LBC-R and MODS-1/LBC-R. In the case of the former, the telescope is configured in binocular mode, and the TCS waits to receive a preset sent from each instrument before moving to the field. Once there, LUCI-1 acquisitions are done normally (now using the RTD interface) and the script can dither as needed by nearIR observations, while LBC-R can either stare or dither. In the case of MODS-1/LBC-R, the telescope is set 


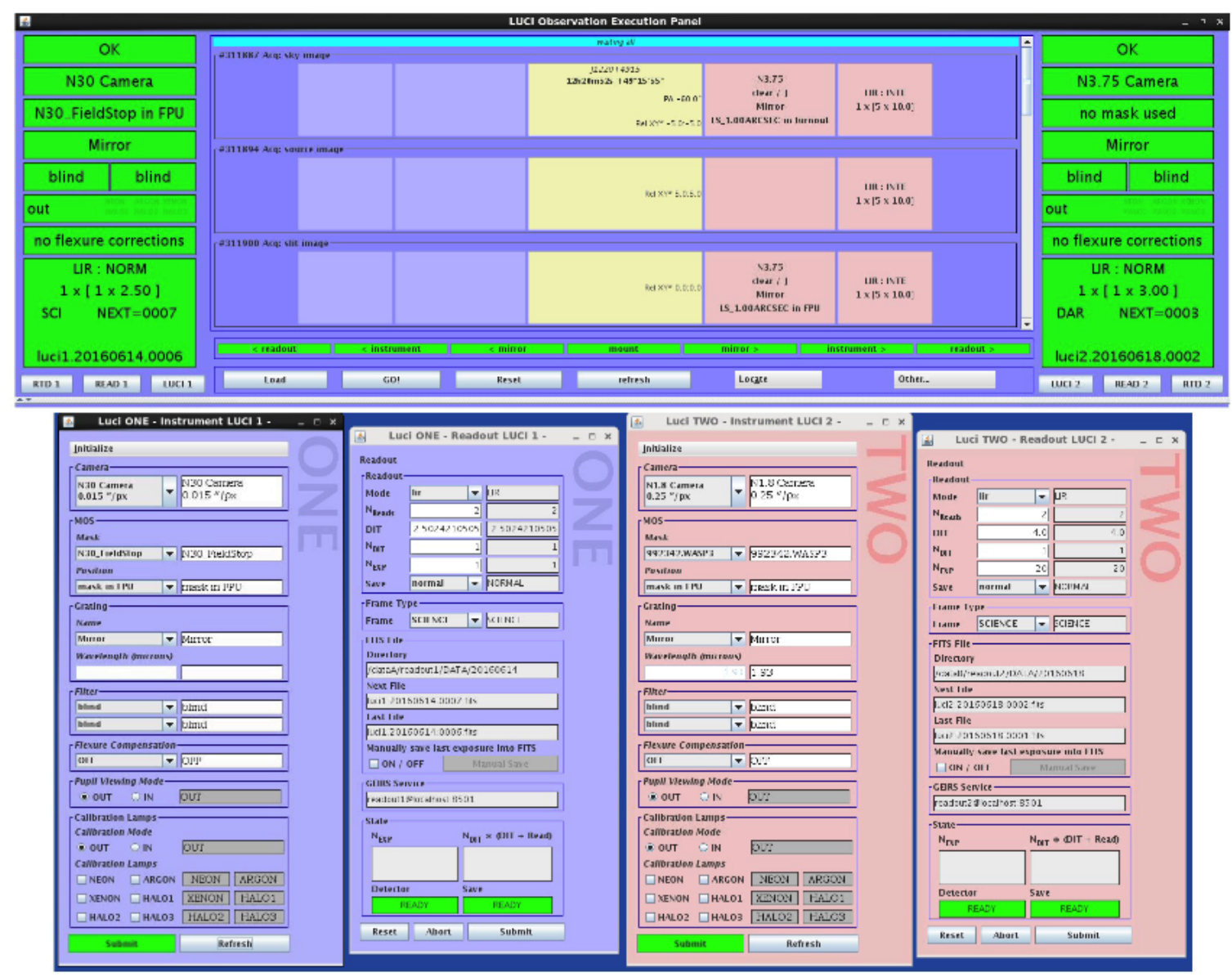

Figure 6: The new LUCI software interface. (Top) The main Observer GUI where scripts are loaded and information about the status of the observations are presented. (Bottom) Readout and Instrument panels for both LUCIs which give the users more manual control over the instrument.

up in a hybrid configuration called "pseudo-monocular." MODS-1 "drives" the mount, i.e. the preset is sent only by MODS- 1 while LBC-R is "along for the ride." On the MODS side, the acquisition is sent normally using the acqMODS Perl script. Once the preset is successful, and the telescope is at the target field, the LBC$\mathrm{R}$ is collimated using DOFPIA and then a modified LBC script is executed that includes a value of $-90^{\circ}$ in the Declination coordinate, which is interpreted by the TCS as a flag to ignore the preset. Alignment and acquisition proceed as normal with modsAlign and the observations are started using the Perl script execMODS. Both sides can guide independently. Dithering can be done using the primary mirrors. Thus, both the dominant (in this case MODS) and the passive side (in this case LBC-R) can dither independently via the mirror. In normal LBC binocular mode, any dithering is done by the mount (which is faster), and not by the two mirrors. However, it was found that in pseudo-monocular mode when LBC (either Red or Blue) dithers using the mirror (which is slower than the mount), the start of the exposure does not wait for the slower move and collimation update from the primary mirror. Work to correct this is underway. Figure 8 plots the limits of the M1 (primary) and M2 (secondary) on SX (configured with MODS-1) with M1 on DX configured with LBC Red. The plot shows the co-pointing limits and how they are affected by the motions available to M1 and M2 together.

With LUCI-1 off the telescope, and with MODS-1 unavailable in November 2015, Mixed-Mode has been successfully tested with MODS-2/LBC-B and LUCI-2/LBC-B. As of this writing, LBTO has not tested a MixedMode LUCI/MODS combination. Based on current limitations, it is theoretically possible this mode should work in "pseudo-monocular" mode. Since LUCI observations (imaging and spectroscopy) require dithering, the most 


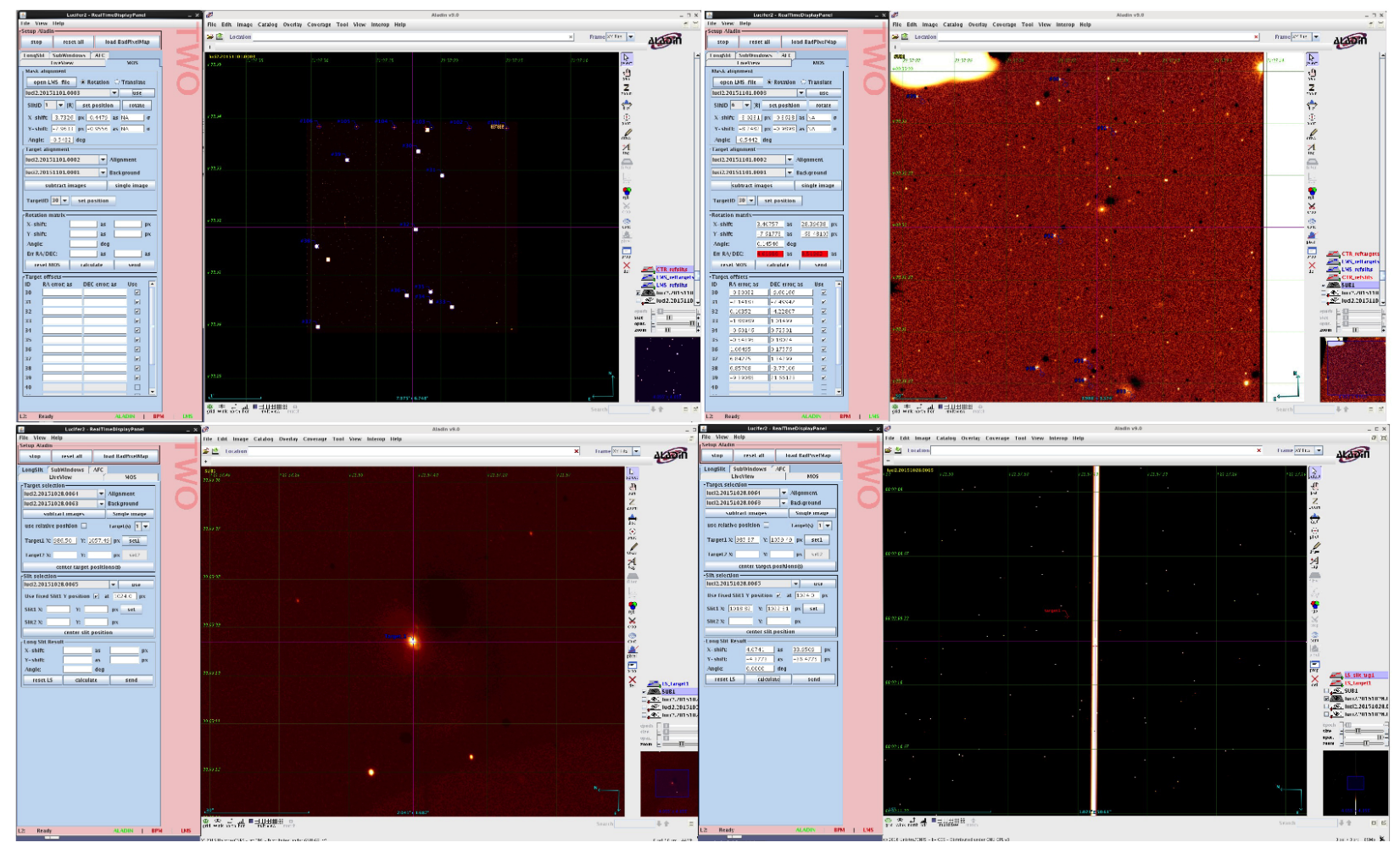

Figure 7: The Real Time Display showing example of MOS mask acquisition (Top) and longslit acquisition (Bottom).

effective combination is LUCI as the dominant instrument and MODS in passive mode. This mode is scheduled to be tested in the near future.

\section{NON-SIDEREAL GUIDING}

Non-sidereal guiding for all three facility instruments is accomplished using the NSIGUI (Non-Sidereal Instrument Graphical User Interface). Figure 9 shows the NSIGUI panels and all three tabs (left to right). The simplest method is to either load a properly formatted ephemeris, or to search for the non-sidereal target and retrieve the ephemeris using the JPL HORIZONS database (web interface: \protect|http://ssd.jpl.nasa.gov/horizons.cgi). In cases where an ephemeris does not exist, the middle tab can be used to enter the information on UT time, celestial coordinates, and rates manually. The user then selects the "SET" button in the IIF Non-Sidereal Override Control which sets an override flag in the TCS. Any preset that is sent from LBC, LUCI, or MODS is then overriden or "hijacked" by the non-sidereal coordinates from the NSIGUI. Instrument configurations are not affected by the GUI. Guiding and tracking then proceeds at a non-sidereal rate. The third tab allows users to update the rates as might be needed during observations. Non-sidereal observations using the LBCs have been done on a regular basis for several semesters. Non-sidereal guiding with LUCI and MODS have been tested at rates up to $\sim 100^{\prime \prime}$ hour $^{-1}$, thus making it possible to obtain spectra of non-sidereal targets.

\section{SUMMARY}

Although it was in 2014 that all of the facility instruments arrived and were installed on the telescope, it has been 2015 and 2016A that have been truly exciting. For the first time, all facility instruments have been used on-sky to obtain scientific data. Two-thirds of the facility instruments are available and have been used in full binocular mode, and it is expected that full binocular observations will occur for all facility instruments 

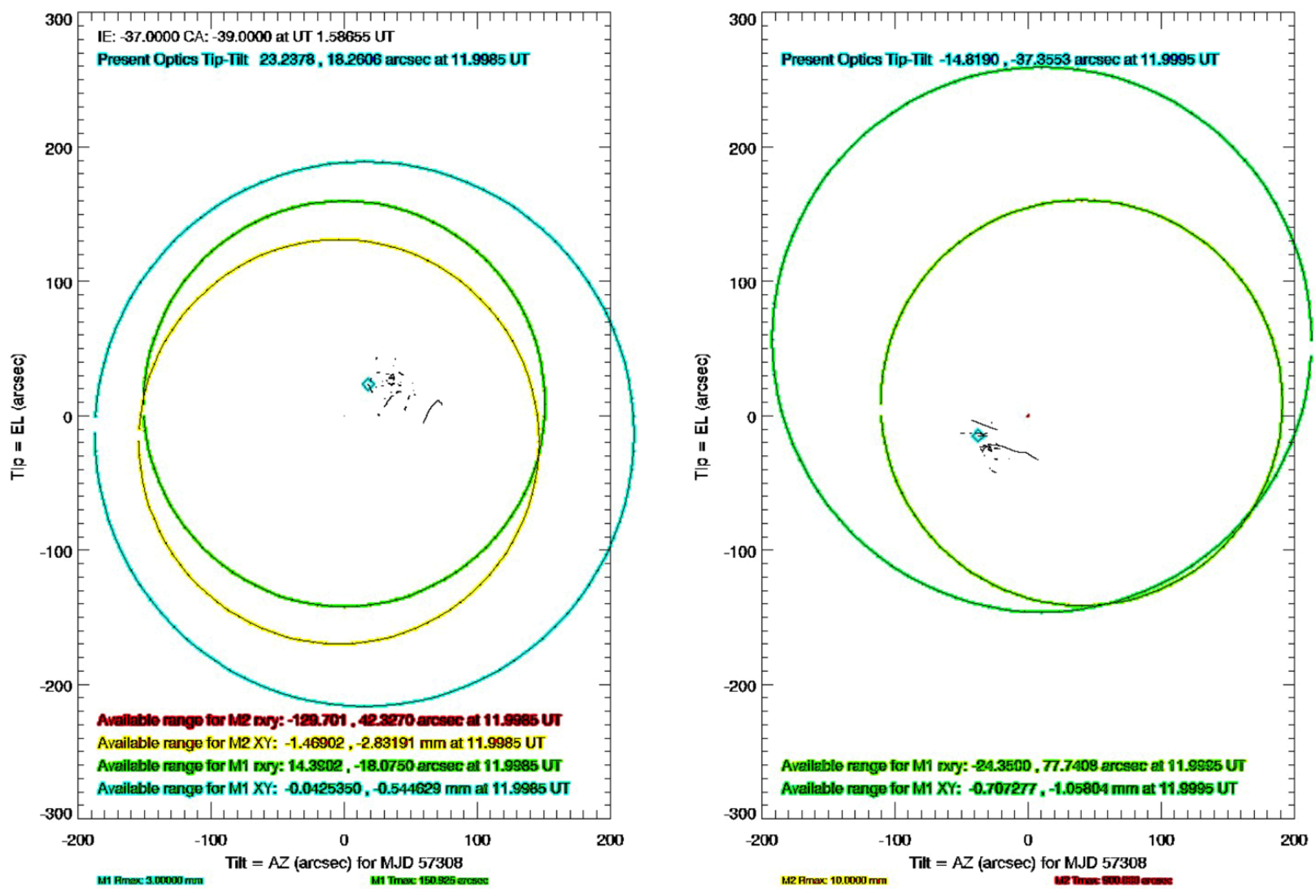

Figure 8: (Left) The co-pointing limits plot for MODS-1 (SX) and (Right) LBC Red (DX) with the telescope configured in pseudo-monocular mode averaged over one hour. The circles indicate the various available ranges of re-pointing available to each side of the telescope. Since LBC Red is at prime focus only M1 is shown on the Right. The small blue diamond represents the current pointing. The XY range of M2 and the tip-tilt range of M1 provide the tightest constraints. The constraints shown here must be considered by PIs in designing their science programs when using Mixed-Mode or Binocular Mode with LUCI or MODS (LBCs dither by moving the mount only, while in Mixed-Mode LBC dithers are done by M1).

in 2016B. Full binocular observations will be a paradigm shift for the nightly operations of LBTO. The parameter space of what LBT can do will significantly expand with full binocular mode, especially in the case of Mixed-Mode observations. Planning tools and scripting tools to address this are already in development and testing, led by the development of queue observing at LBT (see Edwards et al. in the companion proceedings 9910, Observatory Operations: Strategies, Processes, and Systems VI). Full binocular mode will also require a shift in how users propose and design observations to take full advantage of the capabilities of LBT. Information regarding the design and use of instrumentation can be found on the Science Operations webpages: \protecthttp://scienceops.lbto.org/sciops_cookbook/and \protecthttp://abell.as.arizona.edu/ lbtsci/scihome.html as well as the main LBT webpage: www.lbto.org which contains the latest updates and information on all that is happening at the observatory.

\section{ACKNOWLEDGMENTS}

B.Rothberg would like to acknowledge a NASA Keck PI Data Award (Contract \#1462408), administered by JPL and the NASA Exoplanet Science Institute, for support of the work on intermediate redshift ULIRGs. 


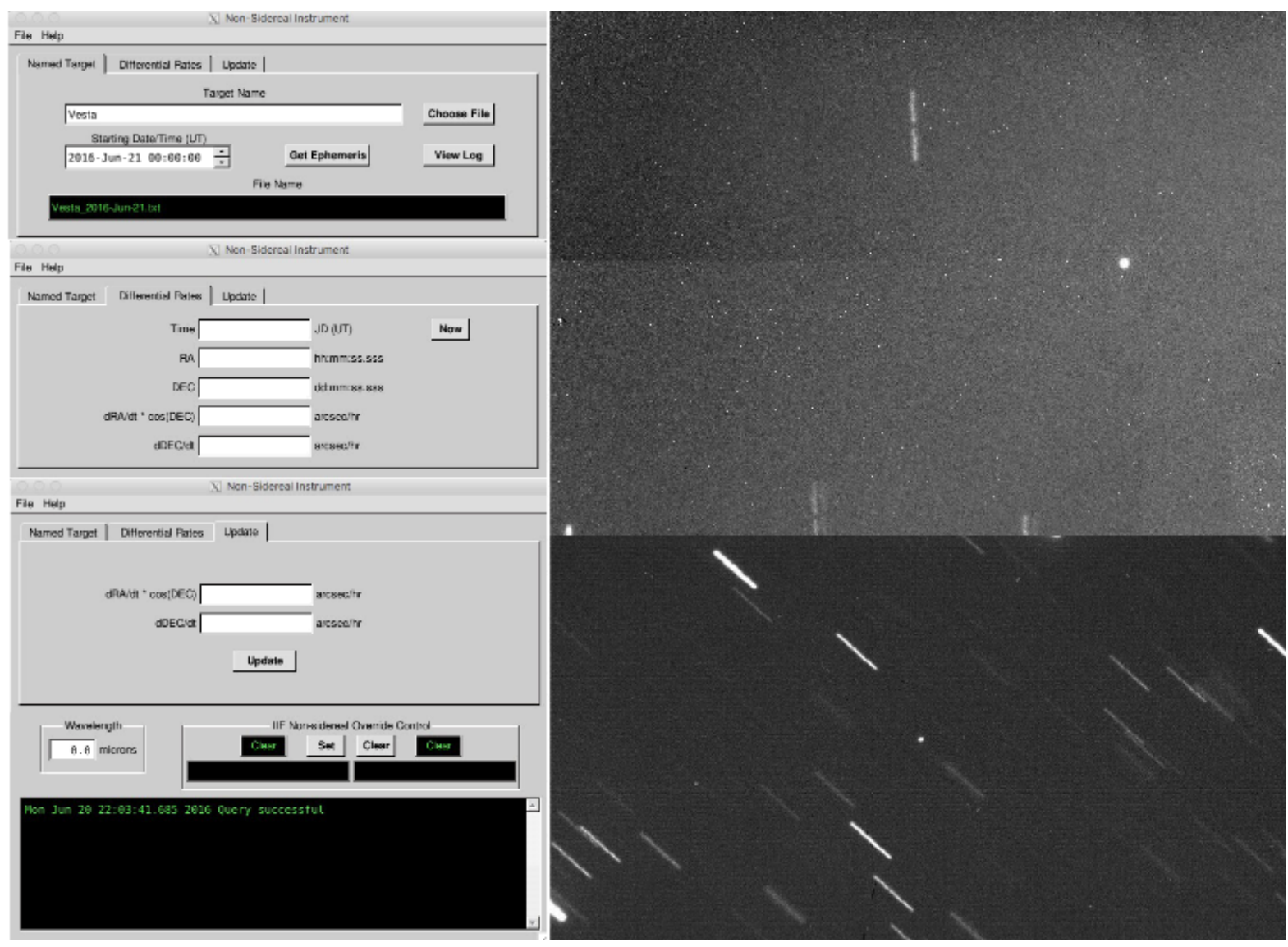

Figure 9: (Left) The three tabs for the NSIGUI (Non-Sidereal Instrument Graphical User Interface). This GUI is used to "hijack" preset coordinates from the facility instruments and replace them with the coordinates determined from an ephemeris or input along with rates in Tab 2. (Top Right) Example of non-sidereal guiding with LUCI-1 of the asteroid Janesick (106" hour $^{-1}$, $300 \mathrm{sec}$ total time on target $-20 \times 15$ sec exposures at z-band) obtained by O. Kuhn. (Bottom Right) LBC Red image of the near-Earth asteroid 2012 ER3 (100" hour $^{-1}, 100$ seconds at Sloan $r$ ) from Hill et al. (2012) $\underline{45}$

\section{REFERENCES}

[1] Hill, J. M. and Salinari, P., "The Large Binocular Telescope project," in [Ground-based Telescopes], Oschmann, Jr., J. M., ed., Proc. SPIE 5489, 603-614 (Oct. 2004).

[2] Ashby, D. S., McKenna, D., Brynnel, J. G., Sargent, T., Cox, D., Little, J., Powell, K., and Holmberg, G., "The Large Binocular Telescope mount control system architecture," in [Society of Photo-Optical Instrumentation Engineers (SPIE) Conference Series], Proc. SPIE 6274, 627423 (June 2006).

[3] Hill, J. M., Green, R. F., and Slagle, J. H., "The Large Binocular Telescope," in [Society of Photo-Optical Instrumentation Engineers (SPIE) Conference Series], Proc. SPIE 6267, 62670Y (June 2006).

[4] Hill, J. M., Green, R. F., Ashby, D. S., Brynnel, J. G., Cushing, N. J., Little, J., Slagle, J. H., and Wagner, R. M., "The Large Binocular Telescope," in [Ground-based and Airborne Telescopes III], Proc. SPIE 7733, $77330 \mathrm{C}$ (July 2010).

[5] Wagner, R. M., Edwards, M. L., Kuhn, O., Thompson, D., and Veillet, C., "An overview and the current status of instrumentation at the Large Binocular Telescope Observatory," in [Ground-based and Airborne Instrumentation for Astronomy V], Proc. SPIE 9147, 914705 (July 2014).

[6] Strassmeier, K. G., Woche, M., Ilyin, I., Popow, E., Bauer, S.-M., Dionies, F., Fechner, T., Weber, M., Hofmann, A., Storm, J., Materne, R., Bittner, W., Bartus, J., Granzer, T., Denker, C., Carroll, T., Kopf, 
M., DiVarano, I., Beckert, E., and Lesser, M., "PEPSI: the Potsdam Echelle Polarimetric and Spectroscopic Instrument for the LBT," in [Ground-based and Airborne Instrumentation for Astronomy II], Proc. SPIE 7014, 70140N (July 2008).

[7] Hinz, P. M., Bippert-Plymate, T., Breuninger, A., Connors, T., Duffy, B., Esposito, S., Hoffmann, W., Kim, J., Kraus, J., McMahon, T., Montoya, M., Nash, R., Durney, O., Solheid, E., Tozzi, A., and Vaitheeswaran, V., "Status of the LBT interferometer," in [Optical and Infrared Interferometry], Proc. SPIE 7013, 701328 (July 2008).

[8] Wilson, J. C., Hinz, P. M., Skrutskie, M. F., Jones, T., Solheid, E., Leisenring, J., Garnavich, P., Kenworthy, M., Nelson, M. J., and Woodward, C. E., "LMIRcam: an L/M-band imager for the LBT combined focus," in [Optical and Infrared Interferometry], Proc. SPIE 7013, 70133A (July 2008).

[9] Skrutskie, M. F., Jones, T., Hinz, P., Garnavich, P., Wilson, J., Nelson, M., Solheid, E., Durney, O., Hoffmann, W., Vaitheeswaran, V., McMahon, T., Leisenring, J., and Wong, A., "The Large Binocular Telescope mid-infrared camera (LMIRcam): final design and status," in [Ground-based and Airborne Instrumentation for Astronomy III], Proc. SPIE 7735, 77353H (July 2010).

[10] Leisenring, J. M., Skrutskie, M. F., Hinz, P. M., Skemer, A., Bailey, V., Eisner, J., Garnavich, P., Hoffmann, W. F., Jones, T., Kenworthy, M., Kuzmenko, P., Meyer, M., Nelson, M., Rodigas, T. J., Wilson, J. C., and Vaitheeswaran, V., "On-sky operations and performance of LMIRcam at the Large Binocular Telescope," in [Ground-based and Airborne Instrumentation for Astronomy IV], Proc. SPIE 8446, 84464F (Sept. 2012).

[11] Hoffmann, W. F., Hinz, P. M., Defrère, D., Leisenring, J. M., Skemer, A. J., Arbo, P. A., Montoya, M., and Mennesson, B., "Operation and performance of the mid-infrared camera, NOMIC, on the Large Binocular Telescope," in [Ground-based and Airborne Instrumentation for Astronomy V], Proc. SPIE 9147, 914710 (July 2014).

[12] Conrad, A., de Kleer, K., Leisenring, J., La Camera, A., Arcidiacono, C., Bertero, M., Boccacci, P., Defrère, D., de Pater, I., Hinz, P., Hofmann, K.-H., Kürster, M., Rathbun, J., Schertl, D., Skemer, A., Skrutskie, M., Spencer, J., Veillet, C., Weigelt, G., and Woodward, C. E., "Spatially Resolved M-band Emission from Io's Loki Patera-Fizeau Imaging at the $22.8 \mathrm{~m}$ LBT," $A J$ 149, 175 (May 2015).

[13] Skemer, A. J., Morley, C. V., Zimmerman, N. T., Skrutskie, M. F., Leisenring, J., Buenzli, E., Bonnefoy, M., Bailey, V., Hinz, P., Defrére, D., Esposito, S., Apai, D., Biller, B., Brandner, W., Close, L., Crepp, J. R., De Rosa, R. J., Desidera, S., Eisner, J., Fortney, J., Freedman, R., Henning, T., Hofmann, K.-H., Kopytova, T., Lupu, R., Maire, A.-L., Males, J. R., Marley, M., Morzinski, K., Oza, A., Patience, J., Rajan, A., Rieke, G., Schertl, D., Schlieder, J., Stone, J., Su, K., Vaz, A., Visscher, C., Ward-Duong, K., Weigelt, G., and Woodward, C. E., "The LEECH Exoplanet Imaging Survey: Characterization of the Coldest Directly Imaged Exoplanet, GJ 504 b, and Evidence for Superstellar Metallicity," ApJ 817, 166 (Feb. 2016).

[14] Gassler, W., Herbst, T. M., Ragazzoni, R., Andersen, D. R., Arcidiacono, C., Baumeister, H., Beckmann, U., Bertram, T., Bizenberger, P., Bohnhardt, H., Diolaiti, E., Eckart, A., Farinato, J., Ligori, S., Rix, H.-W., Rohloff, R.-R., Salinari, P., Soci, R., Straubmeier, C., Vernet-Viard, E., Weigelt, G., Weiss, R., and $\mathrm{Xu}, \mathrm{W} .$, "LINC-NIRVANA: first attempt of an instrument for a 23-m-class telescope," in [Second Backaskog Workshop on Extremely Large Telescopes], Ardeberg, A. L. and Andersen, T., eds., Proc. SPIE 5382, 742-747 (July 2004).

[15] Herbst, T. M., Ragazzoni, R., Eckart, A., and Weigelt, G., "The LINC-NIRVANA high resolution imager: challenges from the lab to first light," in [Ground-based and Airborne Instrumentation for Astronomy V], Proc. SPIE 9147, 91471M (July 2014).

[16] Crepp, J. R., Bechter, A., Bechter, E., Berg, M., Carroll, J., Collins, K., Corpuz, T., Ketterer, R., Kielb, E., Stoddard, R., Eisner, J. A., Gaudi, B. S., Hinz, P., Kratter, K. M., Macela, G., Quirrenbach, A., Skrutskie, M. F., Sozzetti, A., Woodward, C. E., and Zhao, B., "iLocater: A Diffraction-Limited Doppler Spectrometer for the Large Binocular Telescope," in [American Astronomical Society Meeting Abstracts \#223], American Astronomical Society Meeting Abstracts 223, 348.20 (Jan. 2014).

[17] Veillet, C., Brynnel, J., Hill, J., Wagner, R., Ashby, D., Christou, J., Little, J., and Summers, D., "LBTO's long march to full operation - step 1," in [Observatory Operations: Strategies, Processes, and Systems V], Proc. SPIE 9149, 914916 (Aug. 2014). 
[18] Ragazzoni, R., Giallongo, E., Pasian, F., Baruffolo, A., Bertram, R., Diolaiti, E., Di Paola, A., Farinato, J., Gentile, G., Hill, J., Lombini, M., Pedichini, F., Speziali, R., Smareglia, R., and Vernet, E., "The widefield eyes of the Large Binocular Telescope," in [Society of Photo-Optical Instrumentation Engineers (SPIE) Conference Series], Proc. SPIE 6267, 626710 (June 2006).

[19] Speziali, R., Di Paola, A., Giallongo, E., Pedichini, F., Ragazzoni, R., Testa, V., Baruffolo, A., De Santis, C., Diolaiti, E., Farinato, J., Fontana, A., Gallozzi, S., Gasparo, F., Gentile, G., Grazian, A., Manzato, P., Pasian, F., Smareglia, R., and Vernet, E., "The Large Binocular Camera: description and performances of the first binocular imager," in [Ground-based and Airborne Instrumentation for Astronomy II], Proc. SPIE 7014, 70144T (July 2008).

[20] Giallongo, E., Ragazzoni, R., Grazian, A., Baruffolo, A., Beccari, G., de Santis, C., Diolaiti, E., di Paola, A., Farinato, J., Fontana, A., Gallozzi, S., Gasparo, F., Gentile, G., Green, R., Hill, J., Kuhn, O., Pasian, F., Pedichini, F., Radovich, M., Salinari, P., Smareglia, R., Speziali, R., Testa, V., Thompson, D., Vernet, E., and Wagner, R. M., "The performance of the blue prime focus large binocular camera at the large binocular telescope," A\&\&A 482, 349-357 (Apr. 2008).

[21] Wynne, C. G., "Improved three-lens field correctors for paraboloids," MNRAS 160, 13P (1972).

[22] Rochais, T. B., Rothberg, B., and Kuhn, O., "Are the Youngsters Home? A Search for Young Clusters in the Merger Remnant NGC 2655," in [American Astronomical Society Meeting Abstracts], American Astronomical Society Meeting Abstracts 227, 240.12 (Jan. 2016).

[23] Hill, J. M., Ragazzoni, R., Baruffolo, A., Biddick, C. J., Kuhn, O. P., Diolaiti, E., Thompson, D., and Rakich, A., "Prime focus active optics with the Large Binocular Telescope," in [Ground-based and Airborne Telescopes II], Proc. SPIE 7012, 70121M (July 2008).

[24] Wilson, R. N., [Reflecting Telescope Optics II] (1999).

[25] Stangalini, M., Pedichini, F., and Giallongo, title = "Technical Report: LBC Wavefront Reconstructio Software upgrade", i. . I. m. . a. y. . p. . . tech. rep.

[26] Summers, K. R., Di Paola, A., Centrone, M., Edwards, M. L., Hill, J. M., Kuhn, O. P., Pedichini, F., and Summers, D. M., "LBT prime focus camera (LBC) control software upgrades," in [Software and Cyberinfrastructure for Astronomy III], Proc. SPIE 9152, 91522E (July 2014).

[27] Pogge, R. W., Atwood, B., Belville, S. R., Brewer, D. F., Byard, P. L., DePoy, D. L., Derwent, M. A., Eastwood, J., Gonzalez, R., Krygier, A., Marshall, J. R., Martini, P., Mason, J. A., O’Brien, T. P., Osmer, P. S., Pappalardo, D. P., Steinbrecher, D. P., Teiga, E. J., and Weinberg, D. H., "The multi-object double spectrographs for the Large Binocular Telescope," in [Society of Photo-Optical Instrumentation Engineers (SPIE) Conference Series], Proc. SPIE 6269, 62690I (June 2006).

[28] Pogge, R. W., Atwood, B., Brewer, D. F., Byard, P. L., Derwent, M. A., Gonzalez, R., Martini, P., Mason, J. A., O’Brien, T. P., Osmer, P. S., Pappalardo, D. P., Steinbrecher, D. P., Teiga, E. J., and Zhelem, R., "The multi-object double spectrographs for the Large Binocular Telescope," in [Ground-based and Airborne Instrumentation for Astronomy III], Proc. SPIE 7735, 77350A (July 2010).

[29] Pogge, R. W., Atwood, B., O'Brien, T. P., Byard, P. L., Derwent, M. A., Gonzalez, R., Martini, P., Mason, J. A., Osmer, P. S., Pappalardo, D. P., Zhelem, R., Stoll, R. A., Steinbrecher, D. P., Brewer, D. F., Colarosa, C., and Teiga, E. J., "On-sky performance of the Multi-Object Double Spectrograph for the Large Binocular Telescope," in [Ground-based and Airborne Instrumentation for Astronomy IV], Proc. SPIE 8446, 84460G (Sept. 2012).

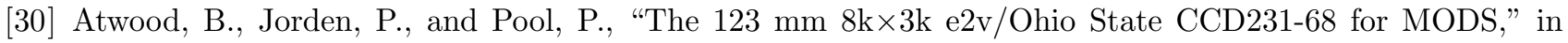
[High Energy, Optical, and Infrared Detectors for Astronomy III], Proc. SPIE 7021, 702108 (July 2008).

[31] Marshall, J. L., O’Brien, T. P., Atwood, B., Byard, P. L., DePoy, D. L., Derwent, M., Eastman, J. D., Gonzalez, R., Pappalardo, D. P., and Pogge, R. W., "An image motion compensation system for the multiobject double spectrograph," in [Society of Photo-Optical Instrumentation Engineers (SPIE) Conference Series], Proc. SPIE 6269, 62691J (June 2006).

[32] Reynolds, R. O., Derwent, M., Power, J., Kuhn, O., Thompson, D., O'Brien, T. P., Pogge, R. W., and Wagner, R. M., "The instrument focal plane mask program at the Large Binocular Telescope," in [Advances in Optical and Mechanical Technologies for Telescopes and Instrumentation], Proc. SPIE 9151, 91514B (July 2014). 
[33] Rothberg, B., Fischer, J., Rodrigues, M., and Pirzkal, N., "A Monster At Any Other Epoch: Are Intermediate Redshift ULIRGs the Progenitors of QSO Host Galaxies?," IAU General Assembly 22, 2257946 (Aug. 2015).

[34] Sanders, D. B., Soifer, B. T., Elias, J. H., Madore, B. F., Matthews, K., Neugebauer, G., and Scoville, N. Z., "Ultraluminous infrared galaxies and the origin of quasars," ApJ 325, 74-91 (Feb. 1988).

[35] Rothberg, B., Fischer, J., Rodrigues, M., and Sanders, D. B., "Unveiling the $\sigma$-discrepancy. II. Revisiting the Evolution of ULIRGs and the Origin of Quasars," ApJ 767, 72 (Apr. 2013).

[36] Seifert, W., Appenzeller, I., Baumeister, H., Bizenberger, P., Bomans, D., Dettmar, R.-J., Grimm, B., Herbst, T., Hofmann, R., Juette, M., Laun, W., Lehmitz, M., Lemke, R., Lenzen, R., Mandel, H., Polsterer, K., Rohloff, R.-R., Schuetze, A., Seltmann, A., Thatte, N. A., Weiser, P., and Xu, W., "LUCIFER: a Multi-Mode NIR Instrument for the LBT," in [Instrument Design and Performance for Optical/Infrared Ground-based Telescopes], Iye, M. and Moorwood, A. F. M., eds., Proc. SPIE 4841, 962-973 (Mar. 2003).

[37] Ageorges, N., Seifert, W., Jütte, M., Knierim, V., Lehmitz, M., Germeroth, A., Buschkamp, P., Polsterer, K., Pasquali, A., Naranjo, V., Gemperlein, H., Hill, J., Feiz, C., Hofmann, R., Laun, W., Lederer, R., Lenzen, R., Mall, U., Mandel, H., Müller, P., Quirrenbach, A., Schäffner, L., Storz, C., and Weiser, P., "LUCIFER1 commissioning at the LBT," in [Ground-based and Airborne Instrumentation for Astronomy III], Proc. SPIE 7735, 77351L (July 2010).

[38] Buschkamp, P., Seifert, W., Polsterer, K., Hofmann, R., Gemperlein, H., Lederer, R., Lehmitz, M., Naranjo, V., Ageorges, N., Kurk, J., Eisenhauer, F., Rabien, S., Honsberg, M., and Genzel, R., "LUCI in the sky: performance and lessons learned in the first two years of near-infrared multi-object spectroscopy at the LBT," in [Ground-based and Airborne Instrumentation for Astronomy IV], Proc. SPIE 8446, 84465L (Sept. 2012).

[39] Rabien, S., Ageorges, N., Barl, L., Beckmann, U., Blümchen, T., Bonaglia, M., Borelli, J. L., Brynnel, J., Busoni, L., Carbonaro, L., Davies, R., Deysenroth, M., Durney, O., Elberich, M., Esposito, S., Gasho, V., Gässler, W., Gemperlein, H., Genzel, R., Green, R., Haug, M., Hart, M. L., Hubbard, P., Kanneganti, S., Masciadri, E., Noenickx, J., Orban de Xivry, G., Peter, D., Quirrenbach, A., Rademacher, M., Rix, H. W., Salinari, P., Schwab, C., Storm, J., Strüder, L., Thiel, M., Weigelt, G., and Ziegleder, J., "ARGOS: the laser guide star system for the LBT," in [Adaptive Optics Systems II], Proc. SPIE 7736, 77360E-77360E-12 (July 2010).

[40] Rabien, S., Barl, L., Beckmann, U., Bonaglia, M., Borelli, J. L., Brynnel, J., Buschkamp, P., Busoni, L., Christou, J., Connot, C., Davies, R., Deysenroth, M., Esposito, S., Gässler, W., Gemperlein, H., Hart, M., Kulas, M., Lefebvre, M., Lehmitz, M., Mazzoni, T., Nussbaum, E., Orban de Xivry, G., Peter, D., Quirrenbach, A., Raab, W., Rahmer, G., Storm, J., and Ziegleder, J., "Status of the ARGOS project," in [Adaptive Optics Systems IV], Proc. SPIE 9148, 91481B (July 2014).

[41] Rahmer, G., Lefebvre, M., Christou, J., Raab, W., Rabien, S., Ziegleder, J., Borelli, J. L., and Gässler, W., "Early laser operations at the Large Binocular Telescope Observatory," in [Observatory Operations: Strategies, Processes, and Systems V], Proc. SPIE 9149, 91492A (Aug. 2014).

[42] Hofmann, R., Gemperlein, H., Grimm, B., Jutte, M., Mandel, H., Polsterer, K., and Weisz, H., "The cryogenic MOS unit for LUCIFER," in [Ground-based Instrumentation for Astronomy], Moorwood, A. F. M. and Iye, M., eds., Proc. SPIE 5492, 1243-1253 (Sept. 2004).

[43] Buschkamp, P., Hofmann, R., Gemperlein, H., Polsterer, K., Ageorges, N., Eisenhauer, F., Lederer, R., Honsberg, M., Haug, M., Eibl, J., Seifert, W., and Genzel, R., "The LUCIFER MOS: a full cryogenic mask handling unit for a near-infrared multi-object spectrograph," in [Ground-based and Airborne Instrumentation for Astronomy III], Proc. SPIE 7735, 773579 (July 2010).

[44] Hill, J. M., Ashby, D. S., Brynnel, J. G., Christou, J. C., Little, J. K., Summers, D. M., Veillet, C., and Wagner, R. M., "The Large Binocular Telescope: binocular all the time," in [Ground-based and Airborne Telescopes V], Proc. SPIE 9145, 914502 (July 2014).

[45] Hill, J. M., Green, R. F., Ashby, D. S., Brynnel, J. G., Cushing, N. J., Little, J. K., Slagle, J. H., and Wagner, R. M., "The Large Binocular Telescope," in [Ground-based and Airborne Telescopes IV], Proc. SPIE 8444, 84441A (Sept. 2012). 